\title{
Controlling stability of Bose-Einstein condensation of interacting magnons in an antiferromagnet by an external magnetic field
}

\author{
Naoya Arakawa* \\ Department of Physics, Toho University, Funabashi, Chiba, 274-8510, Japan
}

(Dated: January 9, 2019)

\begin{abstract}
We propose a mechanism for destabilizing or stabilizing Bose-Einstein condensation (BEC) of interacting magnons in an antiferromagnet. We study how the interaction between magnons affects the magnon BEC in a two-sublattice antiferromagnet without and with an external magnetic field. We show that in the absence of the magnetic field the magnon BEC is destabilized by the attractive interband interaction, which is larger than the repulsive intraband interaction. We also show that in the presence of the magnetic field the magnon BEC is stabilized only if the magnetic field is large enough to make the intraband interaction larger than the interband interaction. Our results provide the first step for understanding the interaction effects on the magnon BEC in antiferromagnets and may open interaction physics of multicomponent BEC of magnons in magnets.
\end{abstract}

\section{INTRODUCTION}

Stability of Bose-Einstein condensation (BEC) depends on the sign of the interaction between bosons ${ }^{1}$. $\mathrm{BEC}$ is the phenomenon that many bosons occupy the lowest-energy state ${ }^{23}$. This can be understood in terms of noninteracting bosons in principle. However, if the interaction is attractive, the BEC becomes unstable; if the interaction is repulsive, the BEC remains stable ${ }^{4}$. Thus whether the interaction is attractive or repulsive is vital for understanding the stability of the BEC.

Nevertheless, it is unclear how the interaction between magnons affects the BEC of magnons in an antiferromagnet. Since magnons are bosonic quasiparticles of a magnetically ordered system, the BEC of magnons is expected to occur in magnets 10 . In addition, theoretical work has showen that the magnon BEC in ferrimagnets and ferromagnets remains stable even in the presence of the magnon-magnon interaction as long as the magnets have a sublattice structure ${ }^{11}$. However, this result is not directly applicable to an antiferromagnet due to the following essential difference: low-energy magnons of an antiferromagnet have band degeneracy 12 , whereas lowenergy magnons of a ferrimagnet or a ferromagnet are described by a single band ${ }^{11|13| 14}$. Because of this difference the magnon BEC for an antiferromagnet gets a multicomponent order parameter; the order parameter for a ferrimagnet or ferromagnet is one-component 1115 . Then this multicomponent $\mathrm{BEC}$ can be converted into the one-component BEC by using an external magnetic field that lifts the band degeneracy. These properties indicate that an antiferromagnet provides new opportunities to study the multicomponent BEC of magnons and the unique magnetic-field effect. Nevertheless, the stability against the interaction remains unclear.

In this paper we study the BEC of interacting magnons in a two-sublattice antiferromagnet and show a mechanism for controlling its stability. Our antiferromagnet is described by a spin Hamiltonian consisting of the antiferromagnetic Heisenberg interaction between nearestneighbor spins, the single-ion anisotropy, and the exter- nal magnetic field. After expressing this Hamiltonian in terms of magnon operators and remarking on several properties, we formulate an effective theory of the BEC of interacting magnons for the antiferromagnet and then study the stability in the absence and the presence of the external magnetic field. We show that the magnon $\mathrm{BEC}$ is unstable in the absence of the external magnetic field due to the attractive interband interaction, which is larger than the repulsive intraband interaction. We also show that when the external magnetic field exceeds a critical value, the repulsive intraband interaction becomes larger than the attractive interband interaction, and thus the magnon BEC remains stable. The main results are summarized in Table I.

We believe that our theory can study the interaction effects on the stability of the BEC of quasiequilibrium magnons in the antiferromagnet. For realizing the magnon BEC, it is necessary to adjust the chemical potential of a magnon, $\mu$, so that $\mu$ satisfies $\epsilon_{\min }-\mu=0$, where $\epsilon_{\min }$ represents the lowest energy of magnon bands. This can be realized, for example, using an external pumping ${ }^{6}$. Although in the presence of the external pumping the system is generally nonequilibrium, it is possible to realize the quasiequilibrium state in which the distribution of a magnon can be well approximated by the Bose distribution function with finite $\mu$; in such a quasiequilibrium state the main effect of the external pumping is to change the value of $\mu$. However, even if $\mu$ is adjusted so that $\epsilon_{\min }-\mu=0$ is satisfied for noninteracting magnons, the magnon BEC becomes unstable for the attractive interaction. Since a system at certain times of a quasiequilibrium state can be approximately described by the magnons whose distribution function is the Bose distribution function with finite $\mu$, our theory can study how the interaction affects the stability of the magnon BEC at a snapshot of the quasiequilibrium state. 
TABLE I. Magnon properties of the antiferromagnet without and with the external magnetic field. Magnon bands are degenerate only for $h=0$. The order parameter of the magnon BEC for $h=0$ is two-component, whereas that for $h \neq 0$ is one-component. The interaction between magnons consists of the intraband interaction and the interband interaction, which are repulsive and attractive, respectively. If the external magnetic field satisfies $|h|>h_{\mathrm{c}}$, the repulsive intraband interaction is larger and the magnon BEC remains stable; otherwise, the attractive interband interaction is larger and the magnon BEC becomes unstable.

\begin{tabular}{cccc}
\hline & $h=0$ & $|h|<h_{\mathrm{c}}$ & $|h|>h_{\mathrm{c}}$ \\
\hline Band degeneracy & Degenerate & Nondegenerate & Nondegenerate \\
Order parameter & Two-component & One-component & One-component \\
Intraband interaction & Repulsive & Repulsive & Repulsive \\
Interband interaction & Attractive & Attractive & Attractive \\
Larger interaction & Interband & Unstable & Intraband \\
Stability of the magnon BEC & Unstable & Stable \\
\hline \hline
\end{tabular}

\section{HAMILTONIAN}

Our antiferromagnet is described by the following spin Hamiltonian:

$$
H=2 J \sum_{\langle\boldsymbol{i}, \boldsymbol{j}\rangle} \boldsymbol{S}_{\boldsymbol{i}} \cdot \boldsymbol{S}_{\boldsymbol{j}}-K \sum_{\boldsymbol{l}}\left(S_{\boldsymbol{l}}^{z}\right)^{2}-h \sum_{\boldsymbol{l}} S_{\boldsymbol{l}}^{z},
$$

where the first term represents the antiferromagnetic Heisenberg interaction between nearest-neighbor spins, the second term $(K>0)$ represents the uniaxial anisotropy energy of a spin for $S>1 / 2$, and the third term represents the Zeeman energy of the external magnetic field; $\sum_{\langle\boldsymbol{i}, \boldsymbol{j}\rangle}$ is the sum for nearest-neighbor spins for $\boldsymbol{i} \in A$ and $\boldsymbol{j} \in B$, where $A$ and $B$ denote $A$ and $B$ sublattices, and $\sum_{l}$ is the sum over all sites; and the number of each sublattice is a half of the number of sites $N$. The above Hamiltonian is a simple model of a collinear antiferomagnet for $\left\langle\boldsymbol{S}_{\boldsymbol{i} \in A}\right\rangle={ }^{t}\left(\begin{array}{lll}0 & 0 & S\end{array}\right)$ and $\left\langle\boldsymbol{S}_{\boldsymbol{j} \in B}\right\rangle={ }^{t}\left(\begin{array}{lll}0 & 0 & -S\end{array}\right)^{16}$. Actually, the antiferromagnetically ordered state of $\mathrm{MnF}_{2}$ can be well described by the spin Hamiltonian consisting of the nearest-neighbor antiferromagnetic Heisenberg interaction with the small anisotropy 17 ; the estimated value is $K / J \approx 0.1$. We thus believe that our model is applicable to $\mathrm{MnF}_{2}$ in the presence of the external magnetic field. Hereafter we consider cases for $J \gg K, h$.

We have considered not only the Heisenberg interaction but also the Zeeman energy and the uniaxial anisotropy energy because the effects of the latter terms are important in antiferromagnets even for $J \gg K, h$. In the ferrimagnet studied in Ref. 11 we did not consider the latter terms because their effects are less important than in antiferromagnets. This difference arises from the following difference in the magnon bands: in the ferrimagnet, the magnon bands are split even for $h=0$ and the energy splitting is typically of order $J$; in the antiferromagnet, the magnon bands are split only for $h \neq 0$ and the energy splitting is of order $h$ [see Eq. (7)]. Since the main effect of the $h$ is to change the value of the energy splitting of magnon bands, its effect for the ferrimagnet is negligible compared with that for the antiferromagnet. Then the uniaxial anisotropy is necessary to keep the magnon energies for $h \neq 0$ nonnegative.
We can express the above Hamiltonian in terms of magnon operators by using the Holstein-Primakoff transformation 1418 . This transformation is expressed as

$$
\begin{gathered}
S_{i}^{z}=S-a_{i}^{\dagger} a_{i}, S_{i}^{-}=a_{i}^{\dagger} \sqrt{2 S-a_{i}^{\dagger} a_{i}}, S_{i}^{+}=\left(S_{i}^{-}\right)^{\dagger}, \\
S_{j}^{z}=-S+b_{j}^{\dagger} b_{j}, S_{j}^{+}=b_{j}^{\dagger} \sqrt{2 S-b_{j}^{\dagger} b_{j}}, S_{j}^{-}=\left(S_{j}^{+}\right)^{\dagger}
\end{gathered}
$$

where $a_{i}^{\dagger}$ and $a_{i}$ are the creation and annihilation operators of a magnon for $i \in A$, and $b_{j}^{\dagger}$ and $b_{j}$ are those of a magnon for $j \in B$. By substituting these equations into Eq. (1), we obtain the magnon Hamiltonian. Since our aim is to clarify the interaction effects on the magnon BEC, stabilized by the kinetic energy, we consider the kinetic energy terms and the dominant terms of the magnon-magnon interaction. These terms can be derived from the standard calculation ${ }^{11 / 13 / 19}$ (for the details see Appendix A). As a result, the kinetic energy terms are given by the following quadratic terms:

$$
H_{\mathrm{KE}}=\sum_{\boldsymbol{q}}\left(a_{\boldsymbol{q}}^{\dagger} b_{\boldsymbol{q}}\right)\left(\begin{array}{cc}
A(\boldsymbol{q})+h & C(\boldsymbol{q}) \\
C(\boldsymbol{q}) & A(\boldsymbol{q})-h
\end{array}\right)\left(\begin{array}{c}
a_{\boldsymbol{q}} \\
b_{\boldsymbol{q}}^{\dagger}
\end{array}\right),
$$

where $a_{\boldsymbol{q}}=\sqrt{\frac{2}{N}} \sum_{\boldsymbol{i}} e^{-i \boldsymbol{q} \cdot \boldsymbol{i}} a_{\boldsymbol{i}}, b_{\boldsymbol{q}}^{\dagger}=\sqrt{\frac{2}{N}} \sum_{\boldsymbol{j}} e^{-i \boldsymbol{q} \cdot \boldsymbol{j}} b_{\boldsymbol{j}}^{\dagger}$, $A(\boldsymbol{q})=2 S[J(\mathbf{0})+K], C(\boldsymbol{q})=2 S J(\boldsymbol{q}), J(\boldsymbol{q})=$ $J \sum_{\delta} e^{i \boldsymbol{q} \cdot \boldsymbol{\delta}}$, and $\boldsymbol{\delta}$ is a vector to nearest neighbors. The interaction terms are then given by the following quartic terms:

$$
\begin{aligned}
H_{\mathrm{int}} & =-\frac{2}{N} \sum_{\boldsymbol{q}, \boldsymbol{q}^{\prime}}\left[J(\mathbf{0}) a_{\boldsymbol{q}}^{\dagger} a_{\boldsymbol{q}} b_{\boldsymbol{q}^{\prime}}^{\dagger} b_{\boldsymbol{q}^{\prime}}+J\left(\boldsymbol{q}-\boldsymbol{q}^{\prime}\right) a_{\boldsymbol{q}}^{\dagger} a_{\boldsymbol{q}^{\prime}} b_{\boldsymbol{q}^{\dagger}}^{\dagger} b_{\boldsymbol{q}^{\prime}}\right. \\
& \left.+J(\boldsymbol{q}) a_{\boldsymbol{q}} b_{\boldsymbol{q}^{\prime}}^{\dagger} b_{\boldsymbol{q}} b_{\boldsymbol{q}^{\prime}}+J(\boldsymbol{q}) a_{\boldsymbol{q}^{\prime}}^{\dagger} a_{\boldsymbol{q}} a_{\boldsymbol{q}^{\prime}} b_{\boldsymbol{q}}\right]+(\text { H.c. }) .
\end{aligned}
$$

The interaction terms consist of the quartic terms arising from the Heisenberg interaction because we have considered the interaction between magnons at different sites; in Eq. (1) only the first term gives such an interaction.

\section{PROPERTIES OF MAGNONS}

The essential difference between the antiferromagnet and a ferromagnet or a ferrimagnet is degeneracy of 
magnon bands. To see this, we diagonalize Eq. (4). This diagonalization can be performed using the Bogoliubov transformation,

$$
\left(\begin{array}{c}
a_{\boldsymbol{q}} \\
b_{\boldsymbol{q}}^{\dagger}
\end{array}\right)=\left(\begin{array}{cc}
c_{\boldsymbol{q}} & -s_{\boldsymbol{q}} \\
-s_{\boldsymbol{q}} & c_{\boldsymbol{q}}
\end{array}\right)\left(\begin{array}{c}
\alpha_{\boldsymbol{q}} \\
\beta_{\boldsymbol{q}}^{\dagger}
\end{array}\right)
$$

where $c_{\boldsymbol{q}}=\cosh \theta_{\boldsymbol{q}}$ and $s_{\boldsymbol{q}}=\sinh \theta_{\boldsymbol{q}}$. Actually, by substituting Eq. (6) into Eq. (4) and setting $\tanh 2 \theta_{\boldsymbol{q}}=$ $C(\boldsymbol{q}) / A(\boldsymbol{q})$, we obtain

$$
H_{\mathrm{KE}}=\sum_{\boldsymbol{q}}\left(\alpha_{\boldsymbol{q}}^{\dagger} \beta_{\boldsymbol{q}}\right)\left(\begin{array}{cc}
\epsilon(\boldsymbol{q})+h & 0 \\
0 & \epsilon(\boldsymbol{q})-h
\end{array}\right)\left(\begin{array}{c}
\alpha_{\boldsymbol{q}} \\
\beta_{\boldsymbol{q}}^{\dagger}
\end{array}\right),
$$

where $\epsilon(\boldsymbol{q})=\sqrt{A(\boldsymbol{q})^{2}-C(\boldsymbol{q})^{2}}$. Equation (7) shows that the $\alpha$ and $\beta$ bands are degenerate for $h=0$. This property is distinct from a lack of such degeneracy in ferromagnets and ferrimagnets; for example, in a ferrimagnet with a similar sublattice structure two bands are nondegenerate even for $h=0$ 11113. This difference results from the difference in time-reversal symmetry, which holds only for the antiferromagnet for $h=0$. Actually, the external magnetic field, which breaks time-reversal symmetry, lifts the band degeneracy, as seen from Eq. (7).

The above properties result in two unique properties of the magnon BEC in the antiferromagnet. Since $\epsilon(\boldsymbol{q})=2 S \sqrt{[J(\mathbf{0})+K]^{2}-J(\boldsymbol{q})^{2}}, \epsilon(\mathbf{0})$ is the lowest energy. A combination of this and the band degeneracy for $h=0$ indicates that in the magnon BEC for $h=0$ a macroscopic number of magnons occupies the $\boldsymbol{q}=\mathbf{0}$ states of the $\alpha$ and $\beta$ bands. Therefore the magnon BEC in the antiferromagnet for $h=0$ possesses a multicomponent order parameter (i.e., $\left\langle\alpha_{\mathbf{0}}\right\rangle$ and $\left\langle\beta_{\mathbf{0}}^{\dagger}\right\rangle$ ). This is in con- trast with the magnon BEC in a ferromagnet and a ferrimagnet because that is described by a one-component order parameter 11115 . Then, by using the external magnetic field, the magnon BEC in the antiferromagnet can change from multicomponent BEC to one-component BEC as a result of lifting the band degeneracy. For example, for $h<0$ the lowest-energy state is the $\boldsymbol{q}=\mathbf{0}$ state of the $\alpha$ band and the order parameter is $\left\langle\alpha_{\mathbf{0}}\right\rangle$.

\section{INTERACTION EFFECTS ON THE STABILITY OF THE MAGNON BEC}

We now formulate the effective theory of the BEC of interacting magnons in the antiferromagnet for $h=0$. Since we can express the terms in Eq. (5) in terms of the operators of the $\alpha$ and $\beta$ bands by using Eq. (6), we can write $H_{\text {int }}$ as the sum of intraband terms and interband terms, $H_{\text {int }}=H_{\text {intra }}+H_{\text {inter }}$, where $H_{\text {intra }}$ consists of the intraband terms of the $\alpha$ band and of the $\beta$ band and $H_{\text {inter }}$ consists of the interband terms between these bands. The expressions for $H_{\text {intra }}$ and $H_{\text {inter }}$ are derived in Appendix B. Then, since the main effects of the interaction terms can be taken into account in the mean-field approximation $\frac{11|13| 19}{}$, we can approximate the terms of $H_{\text {intra }}$ and $H_{\text {inter }}$ by the mean-field interaction terms. Using these procedures and performing a calculation similar to that for the ferrimagnet $\frac{11}{11}$, we can obtain the leading terms of $H_{\text {intra }}$ and $H_{\text {inter. }}$. First, as we show in Appendix $\mathrm{C}$, the contributions from the mean-field interaction terms for $\boldsymbol{q}=\boldsymbol{q}^{\prime}=\mathbf{0}$, for $\boldsymbol{q}=\mathbf{0}, \boldsymbol{q}^{\prime} \neq \mathbf{0}$, and for $\boldsymbol{q} \neq \mathbf{0}, \boldsymbol{q}^{\prime}=\mathbf{0}$ are zero within the leading order. Then, as we derive in Appendix $\mathrm{D}$, the mean-field interaction terms for $\boldsymbol{q} \neq \mathbf{0}, \boldsymbol{q}^{\prime} \neq \mathbf{0}$ are given as follows:

$$
\begin{aligned}
& H_{\text {intra }}=\frac{1}{N} \sum_{\boldsymbol{q}, \boldsymbol{q}^{\prime} \neq \mathbf{0}} \Gamma_{\mathrm{intra} 1}\left(\boldsymbol{q}, \boldsymbol{q}^{\prime}\right)\left(n_{\boldsymbol{q}^{\prime} \alpha} \alpha_{\boldsymbol{q}}^{\dagger} \alpha_{\boldsymbol{q}}+n_{\boldsymbol{q}^{\prime} \beta} \beta_{\boldsymbol{q}}^{\dagger} \beta_{\boldsymbol{q}}\right), \\
& H_{\mathrm{inter}}=\frac{1}{N} \sum_{\boldsymbol{q}, \boldsymbol{q}^{\prime} \neq \mathbf{0}} \Gamma_{\mathrm{inter} 1}\left(\boldsymbol{q}, \boldsymbol{q}^{\prime}\right)\left(n_{\boldsymbol{q}^{\prime} \beta} \alpha_{\boldsymbol{q}}^{\dagger} \alpha_{\boldsymbol{q}}+n_{\boldsymbol{q}^{\prime} \alpha} \beta_{\boldsymbol{q}}^{\dagger} \beta_{\boldsymbol{q}}\right)+\frac{1}{N} \sum_{\boldsymbol{q} \neq \mathbf{0}} \Gamma_{\mathrm{inter} 2}(\boldsymbol{q})\left(n_{\boldsymbol{q} \beta} \alpha_{\boldsymbol{q}}^{\dagger} \alpha_{\boldsymbol{q}}+n_{\boldsymbol{q} \alpha} \beta_{\boldsymbol{q}}^{\dagger} \beta_{\boldsymbol{q}}\right) \\
& +\frac{1}{N} \sum_{\boldsymbol{q}, \boldsymbol{q}^{\prime} \neq \mathbf{0}} \Gamma_{\mathrm{inter} 3}\left(\boldsymbol{q}, \boldsymbol{q}^{\prime}\right)\left(n_{\boldsymbol{q}^{\prime} \alpha}+n_{\boldsymbol{q}^{\prime} \beta}\right)\left(\alpha_{\boldsymbol{q}} \beta_{\boldsymbol{q}}+\alpha_{\boldsymbol{q}}^{\dagger} \beta_{\boldsymbol{q}}^{\dagger}\right)+\frac{1}{N} \sum_{\boldsymbol{q} \neq \mathbf{0}} \Gamma_{\mathrm{inter} 4}(\boldsymbol{q})\left(n_{\boldsymbol{q} \alpha}+n_{\boldsymbol{q} \beta}\right)\left(\alpha_{\boldsymbol{q}} \beta_{\boldsymbol{q}}+\alpha_{\boldsymbol{q}}^{\dagger} \beta_{\boldsymbol{q}}^{\dagger}\right),
\end{aligned}
$$

where

$$
\begin{aligned}
& \Gamma_{\text {intra } 1}\left(\boldsymbol{q}, \boldsymbol{q}^{\prime}\right)=-4 J(\mathbf{0})\left(c_{\boldsymbol{q}}^{2} s_{\boldsymbol{q}^{\prime}}^{2}+c_{\boldsymbol{q}^{\prime}}^{2} s_{\boldsymbol{q}}^{2}\right)-8 J\left(\boldsymbol{q}-\boldsymbol{q}^{\prime}\right) c_{\boldsymbol{q}} s_{\boldsymbol{q}} c_{\boldsymbol{q}^{\prime}} s_{\boldsymbol{q}^{\prime}}+4 J(\boldsymbol{q}) c_{\boldsymbol{q}} s_{\boldsymbol{q}}\left(c_{\boldsymbol{q}^{\prime}}^{2}+s_{\boldsymbol{q}^{\prime}}^{2}\right)+4 J\left(\boldsymbol{q}^{\prime}\right) c_{\boldsymbol{q}^{\prime}} s_{\boldsymbol{q}^{\prime}}\left(c_{\boldsymbol{q}}^{2}+s_{\boldsymbol{q}}^{2}\right), \\
& \Gamma_{\text {inter } 1}\left(\boldsymbol{q}, \boldsymbol{q}^{\prime}\right)=-4 J(\mathbf{0})\left(c_{\boldsymbol{q}}^{2} c_{\boldsymbol{q}^{\prime}}^{2}+s_{\boldsymbol{q}^{\prime}}^{2} \boldsymbol{q}_{\boldsymbol{q}}^{2}\right)-8 J\left(\boldsymbol{q}-\boldsymbol{q}^{\prime}\right) c_{\boldsymbol{q}} s_{\boldsymbol{q}} c_{\boldsymbol{q}^{\prime}} s_{\boldsymbol{q}^{\prime}}+4 J(\boldsymbol{q}) c_{\boldsymbol{q}} s_{\boldsymbol{q}}\left(c_{\boldsymbol{q}^{\prime}}^{2}+s_{\boldsymbol{q}^{\prime}}^{2}\right)+4 J\left(\boldsymbol{q}^{\prime}\right) c_{\boldsymbol{q}^{\prime}} s_{\boldsymbol{q}^{\prime}}\left(c_{\boldsymbol{q}}^{2}+s_{\boldsymbol{q}}^{2}\right), \\
& \Gamma_{\text {inter } 2}(\boldsymbol{q})=-4 J(\mathbf{0})\left(c_{\boldsymbol{q}}^{2}+s_{\boldsymbol{q}}^{2}\right)^{2}+8 J(\boldsymbol{q}) c_{\boldsymbol{q}} s_{\boldsymbol{q}}\left(c_{\boldsymbol{q}}^{2}+s_{\boldsymbol{q}}^{2}\right) \\
& \Gamma_{\text {inter } 3}\left(\boldsymbol{q}, \boldsymbol{q}^{\prime}\right)=4 J(\mathbf{0}) c_{\boldsymbol{q}} s_{\boldsymbol{q}}\left(c_{\boldsymbol{q}^{\prime}}^{2}+s_{\boldsymbol{q}^{\prime}}^{2}\right)+4 J\left(\boldsymbol{q}-\boldsymbol{q}^{\prime}\right) c_{\boldsymbol{q}^{\prime}} s_{\boldsymbol{q}^{\prime}}\left(c_{\boldsymbol{q}}^{2}+s_{\boldsymbol{q}}^{2}\right)-2 J(\boldsymbol{q})\left(c_{\boldsymbol{q}}^{2}+s_{\boldsymbol{q}}^{2}\right)\left(c_{\boldsymbol{q}^{\prime}}^{2}+s_{\boldsymbol{q}^{\prime}}^{2}\right)-8 J\left(\boldsymbol{q}^{\prime}\right) c_{\boldsymbol{q}} s_{\boldsymbol{q}} c_{\boldsymbol{q}^{\prime}} s_{\boldsymbol{q}^{\prime}} \\
& \Gamma_{\text {inter } 4}(\boldsymbol{q})=8 J(\mathbf{0}) c_{\boldsymbol{q}} s_{\boldsymbol{q}}\left(c_{\boldsymbol{q}}^{2}+s_{\boldsymbol{q}}^{2}\right)-2 J(\boldsymbol{q})\left(c_{\boldsymbol{q}}^{2}+s_{\boldsymbol{q}}^{2}\right)^{2}-8 J(\boldsymbol{q}) c_{\boldsymbol{q}}^{2} s_{\boldsymbol{q}}^{2}
\end{aligned}
$$

In Eqs. (8) and 9 we have defined $n_{\boldsymbol{q} \alpha}=\left\langle\alpha_{\boldsymbol{q}}^{\dagger} \alpha_{\boldsymbol{q}}\right\rangle=$ $n[\epsilon(\boldsymbol{q})]$ and $n_{\boldsymbol{q} \beta}=\left\langle\beta_{\boldsymbol{q}}^{\dagger} \beta_{\boldsymbol{q}}\right\rangle=n[\epsilon(\boldsymbol{q})]$, where $n(\epsilon)$ is the Bose distribution function. Then, since dominant 
low-energy excitations are described by low- $\boldsymbol{q}$ magnons of the $\alpha$ and $\beta$ bands, we can estimate $\Gamma_{\text {intral }}\left(\boldsymbol{q}, \boldsymbol{q}^{\prime}\right)$, $\Gamma_{\text {inter } 1}\left(\boldsymbol{q}, \boldsymbol{q}^{\prime}\right), \Gamma_{\text {inter } 2}(\boldsymbol{q}), \Gamma_{\text {inter } 3}\left(\boldsymbol{q}, \boldsymbol{q}^{\prime}\right)$, and $\Gamma_{\text {inter } 4}(\boldsymbol{q})$ in the limit $|\boldsymbol{q}|,\left|\boldsymbol{q}^{\prime}\right| \rightarrow 0$. After the calculation described in Appendix E, we obtain

$$
\begin{aligned}
& \Gamma_{\text {intra1 }}\left(\boldsymbol{q}, \boldsymbol{q}^{\prime}\right) \sim 2 J(\mathbf{0}), \\
& \Gamma_{\text {inter } 1}\left(\boldsymbol{q}, \boldsymbol{q}^{\prime}\right) \sim-2 J(\mathbf{0}), \\
& \Gamma_{\text {inter } 2}(\boldsymbol{q}) \sim-2 J(\mathbf{0}), \\
& \Gamma_{\text {inter } 3}\left(\boldsymbol{q}, \boldsymbol{q}^{\prime}\right) \sim 0, \\
& \Gamma_{\text {inter } 4}(\boldsymbol{q}) \sim 0 .
\end{aligned}
$$

Thus the leading term of the intraband interaction is repulsive, whereas that of the interband interaction is attractive. In addition, Eqs. (15)-19 with Eqs. (8) and (9) show that the leading terms of $H_{\text {intra }}$ and $H_{\text {inter }}$ are written as follows:

$$
\begin{aligned}
& H_{\text {intra }}=\frac{2}{N} J(\mathbf{0}) \sum_{\boldsymbol{q}, \boldsymbol{q}^{\prime} \neq \mathbf{0}}\left(n_{\boldsymbol{q}^{\prime} \alpha} \alpha_{\boldsymbol{q}}^{\dagger} \alpha_{\boldsymbol{q}}+n_{\boldsymbol{q}^{\prime} \beta} \beta_{\boldsymbol{q}}^{\dagger} \beta_{\boldsymbol{q}}\right) \\
& H_{\text {inter }}=-\frac{2}{N} J(\mathbf{0}) \sum_{\boldsymbol{q}, \boldsymbol{q}^{\prime} \neq \mathbf{0}}\left(n_{\boldsymbol{q}^{\prime} \beta} \alpha_{\boldsymbol{q}}^{\dagger} \alpha_{\boldsymbol{q}}+n_{\boldsymbol{q}^{\prime} \alpha} \beta_{\boldsymbol{q}}^{\dagger} \beta_{\boldsymbol{q}}\right) \\
& -\frac{2}{N} J(\mathbf{0}) \sum_{\boldsymbol{q} \neq \mathbf{0}}\left(n_{\boldsymbol{q} \beta} \alpha_{\boldsymbol{q}}^{\dagger} \alpha_{\boldsymbol{q}}+n_{\boldsymbol{q} \alpha} \beta_{\boldsymbol{q}}^{\dagger} \beta_{\boldsymbol{q}}\right) .
\end{aligned}
$$

Combining Eqs. 20 and 21) with Eq. (7) for $h=0$ and using $n_{\boldsymbol{q} \alpha}=n_{\boldsymbol{q} \beta}=n[\epsilon(\boldsymbol{q})]$, we obtain the following effective Hamiltonian:

$$
H_{\mathrm{eff}}=\sum_{\boldsymbol{q}} \epsilon^{*}(\boldsymbol{q})\left(\alpha_{\boldsymbol{q}}^{\dagger} \alpha_{\boldsymbol{q}}+\beta_{\boldsymbol{q}}^{\dagger} \beta_{\boldsymbol{q}}\right)
$$

where

$$
\epsilon^{*}(\boldsymbol{q})=\epsilon(\boldsymbol{q})+\left(1-\delta_{\boldsymbol{q}, \mathbf{0}}\right) \frac{1}{N} \sum_{\boldsymbol{q}^{\prime} \neq \mathbf{0}} \Gamma_{\mathrm{eff}}\left(\boldsymbol{q}, \boldsymbol{q}^{\prime}\right) n\left[\epsilon\left(\boldsymbol{q}^{\prime}\right)\right]
$$

and

$$
\begin{aligned}
\Gamma_{\mathrm{eff}}\left(\boldsymbol{q}, \boldsymbol{q}^{\prime}\right) & =\Gamma_{\text {intra1 }}\left(\boldsymbol{q}, \boldsymbol{q}^{\prime}\right)+\Gamma_{\text {inter } 1}\left(\boldsymbol{q}, \boldsymbol{q}^{\prime}\right)+\delta_{\boldsymbol{q}^{\prime}, \boldsymbol{q}} \Gamma_{\text {inter2 }}(\boldsymbol{q}) \\
& \sim-2 J(\mathbf{0}) \delta_{\boldsymbol{q}^{\prime}, \boldsymbol{q}}
\end{aligned}
$$

By using the above effective theory, we can show that the attractive interband interaction causes the magnon $\mathrm{BEC}$ for $h=0$ to be unstable. If $\epsilon^{*}(\mathbf{0})=\epsilon(\mathbf{0})$ remains the lowest-energy state, the magnon BEC remains stable even in the presence of the magnon-magnon interaction; otherwise, the magnon BEC becomes unstable. The former condition is satisfied if $\Gamma_{\text {eff }}\left(\boldsymbol{q}, \boldsymbol{q}^{\prime}\right)$ is positive, i.e., the effective interaction is repulsive. Note that $n\left[\epsilon\left(\boldsymbol{q}^{\prime}\right)\right]$ is non-negative. From Eq. (23) with Eq. (24), we can deduce that the effective interaction becomes attractive and it makes the magnon BEC unstable.

In a similar way we analyze the interaction effects on the magnon BEC for $h \neq 0$ and show the condition for the stability. We consider the case for $h<0$ because the case for $h>0$ can be similarly analyzed. For $h<0$ the band degeneracy is lifted and the low-energy magnons are described by the $\alpha$ band. Since the effect of $h$ is to replace the energy dispersions of noninteracting magnons of the $\alpha$ and $\beta$ bands by $\epsilon_{\alpha}(\boldsymbol{q})=\epsilon(\boldsymbol{q})+h$ and $\epsilon_{\beta}(\boldsymbol{q})=\epsilon(\boldsymbol{q})-h$, respectively, we obtain the effective theory for $h \neq 0$ by replacing $\epsilon(\boldsymbol{q})^{\text {'s }}$ for the $\alpha$ and $\beta$ bands in the effective theory for $h=0$ by $\epsilon_{\alpha}(\boldsymbol{q})$ and $\epsilon_{\beta}(\boldsymbol{q})$, respectively; the expressions of $\Gamma_{\text {intra } 1}\left(\boldsymbol{q}, \boldsymbol{q}^{\prime}\right), \Gamma_{\text {inter } 1}\left(\boldsymbol{q}, \boldsymbol{q}^{\prime}\right), \Gamma_{\text {inter } 2}(\boldsymbol{q})$, $\Gamma_{\text {inter } 3}\left(\boldsymbol{q}, \boldsymbol{q}^{\prime}\right)$, and $\Gamma_{\text {inter4 }}(\boldsymbol{q})$ remain unchanged because $c_{\boldsymbol{q}}^{2}, s_{\boldsymbol{q}}^{2}$, and $c_{\boldsymbol{q}} s_{\boldsymbol{q}}$ are independent of $h$ [see Eqs. (C28)(C32)]. Therefore the effective theory for $h<0$ is described by the following Hamiltonian:

$$
H_{\mathrm{eff}}=\sum_{\boldsymbol{q}} \epsilon_{\alpha}^{*}(\boldsymbol{q}) \alpha_{\boldsymbol{q}}^{\dagger} \alpha_{\boldsymbol{q}}
$$

where

$$
\begin{aligned}
& \epsilon_{\alpha}^{*}(\boldsymbol{q})=\epsilon_{\alpha}(\boldsymbol{q})+\left(1-\delta_{\boldsymbol{q}, \mathbf{0}}\right) \frac{2}{N} J(\mathbf{0}) \sum_{\boldsymbol{q}^{\prime} \neq \mathbf{0}} \\
& \times\left\{n\left[\epsilon_{\alpha}\left(\boldsymbol{q}^{\prime}\right)\right]-\left(1+\delta_{\boldsymbol{q}^{\prime}, \boldsymbol{q}}\right) n\left[\epsilon_{\beta}\left(\boldsymbol{q}^{\prime}\right)\right]\right\} .
\end{aligned}
$$

The above effective Hamiltonian includes not only the intraband interaction of the $\alpha$ band but also the interband interaction between the $\alpha$ and $\beta$ bands because the energy difference between these bands is of order $h$, much smaller than $J$. Equation (26) shows that the magnon BEC is unstable for $n\left[\epsilon_{\alpha}\left(\boldsymbol{q}^{\prime}\right)\right]<\left(1+\delta_{\boldsymbol{q}^{\prime}, \boldsymbol{q}}\right) n\left[\epsilon_{\beta}\left(\boldsymbol{q}^{\prime}\right)\right]$, which means that the repulsive intraband interaction is smaller than the attractive interband interaction, whereas the magnon BEC is stable for $n\left[\epsilon_{\alpha}\left(\boldsymbol{q}^{\prime}\right)\right]>\left(1+\delta_{\boldsymbol{q}^{\prime}, \boldsymbol{q}}\right) n\left[\epsilon_{\beta}\left(\boldsymbol{q}^{\prime}\right)\right]$, which means that the repulsive intraband interaction is larger. Since $n(\epsilon)$ is a monotonically decreasing function and an increase of $|h|$ leads to both an increase of $n\left[\epsilon_{\alpha}\left(\boldsymbol{q}^{\prime}\right)\right]=n\left[\epsilon\left(\boldsymbol{q}^{\prime}\right)-|h|\right]$ and a decrease of $n\left[\epsilon_{\beta}\left(\boldsymbol{q}^{\prime}\right)\right]=$ $n\left[\epsilon\left(\boldsymbol{q}^{\prime}\right)+|h|\right]$ at each $\epsilon\left(\boldsymbol{q}^{\prime}\right)$, there should be a critical value of $|h|, h_{\mathrm{c}}$, which is defined as $n\left[\epsilon\left(\boldsymbol{q}^{\prime}\right)-h_{\mathrm{c}}\right]=$ $\left(1+\delta_{\boldsymbol{q}^{\prime}, \boldsymbol{q}}\right) n\left[\epsilon\left(\boldsymbol{q}^{\prime}\right)+h_{\mathrm{c}}\right]$; this can be seen also from estimating the terms in the curly brackets of Eq. (26) to the leading term in $h^{20}$. Therefore the BEC of interacting magnons remains stable for $|h|>h_{\mathrm{c}}$, whereas it is unstable for $|h|<h_{\mathrm{c}}$.

\section{DISCUSSION}

To get a deeper understanding, we compare our results with the results in ferrimagnets and ferromagnets $11 \mid 15$. First, our result for $h=0$ is distinct from the results in ferrimagnets and ferromagnets ${ }^{1115}$ because multicomponent BEC is characteristic of the antiferromagnet. We thus believe that our result is the first step towards understanding the interaction effects in multicompoment BEC of magnons. Second, our result for $|h|>h_{\mathrm{c}}$ is similar to the results in the two-sublattice ferrimagnet and ferromagnet 11 because these show that the onecomponent BEC of interacting magnons remains stable due to the repulsive intraband interaction. The origin of 
this repulsive interaction can be understood in the same manner: the magnons in the different sublattices give the different contributions to the intraband interaction due to the different coefficients in the Bogoliubov transformation Eq. (6). This similarity suggests that the magnon BEC may be experimentally observed for an antiferromagnet with an external magnetic field; this suggestion is consistent with experiments 89 . Third, our result for $|h|<h_{\mathrm{c}}$ contrasts with the results in the two-sublattice ferrimagnet and ferromagnet ${ }^{111}$ because the former shows that the one-component $\mathrm{BEC}$ of interacting magnons is unstable despite the existence of the sublattice structure. This difference arises from the competition between the repulsive intraband interaction and the attractive interband interaction in the antiferromagnet. Note that the interband interaction is non-negligible only if the energy difference between bands is small because $n(\epsilon)$ is large only for small $\epsilon$. Since that energy difference is typically of order $J$ for ferrimagnets and ferromagnets11|13, the interband interaction is negligible for them. Thus the competition between the intraband and interband interactions is characteristic of the antiferromagnet. From the above comparison we conclude that the interaction effects in the antiferromagnet are more complicated due to the band degeneracy for no external magnetic field and the small energy difference between magnon bands.

We now discuss the implications of our results. Our results show that the stability of the BEC of interacting magnons in the antiferromagnet is controllable by tuning the external magnetic field. Therefore our mechanism can be used for further studies of not only the stable BEC of interacting magnons but also the unstable BEC. In particular, our result for $h=0$ provides a unique opportunity for studying the unstable multicomponent BEC of interacting magnons. Since our model is applicable to $\mathrm{MnF}_{2}$, as described in Sec. II, these properties could be observed in the magnon BEC for $\mathrm{MnF}_{2}$. Our results also show that the sign of the effective interaction between magnons of the antiferromagnet is sensitive to the external magnetic field. This is distinct from the expectation that the properties of antiferromagnets are insensitive to magnetic fields 2123 . Therefore this distinction suggests that it is not always correct to neglect the magnetic-field dependence in antiferromagnets. In particular, in cases of the magnon properties that depend on the sign of the effective interaction between magnons, it is crucial to study the magnetic-field dependence. Then our theory can be extended to more complicated magnets. Our study is thus useful for further studies of the one-component and the multicomponent $\mathrm{BEC}$ of interacting magnons in various magnets.

\section{SUMMARY}

In summary we studied the BEC of interacting magnons in the two-sublattice antiferromagnet without and with the external magnetic field and revealed a method to control its stability. We showed that the magnon BEC for no external magnetic field gets the twocomponent order parameter due to the band degeneracy and this two-component BEC is destabilized by the attractive interband interaction, which is larger than the repulsive intraband interaction. We also showed that the two-component $\mathrm{BEC}$ is converted into one-component BEC by the external magnetic field, which lifts the band degeneracy, and this one-component BEC is stabilized only if the external magnetic field exceeds the critical value, above which the repulsive intraband interaction is larger than the attractive interband interaction.

\section{Appendix A: Derivation of Eqs. (4) and (5)}

We derive Eqs. (4) and (5). By substituting Eqs. (2) and (3) into Eq. (1), we can express the Hamiltonian of Eq. (1) in terms of the magnon operators. Since the quadratic terms correspond to the kinetic energy terms and the quartic terms correspond to the interaction terms $\frac{1113 \mid 19}{\text {, }}$, we explain the details of the derivations of the quadratic terms and the quartic terms. First, the quadratic terms are given by

$$
H_{\mathrm{KE}}=2 J S \sum_{\langle\boldsymbol{i}, \boldsymbol{j}\rangle}\left(a_{\boldsymbol{i}}^{\dagger} a_{\boldsymbol{i}}+b_{\boldsymbol{j}}^{\dagger} b_{\boldsymbol{j}}+a_{\boldsymbol{i}} b_{\boldsymbol{j}}+a_{\boldsymbol{i}}^{\dagger} b_{\boldsymbol{j}}^{\dagger}\right)+(2 K S+h) \sum_{\boldsymbol{i}} a_{\boldsymbol{i}}^{\dagger} a_{\boldsymbol{i}}+(2 K S-h) \sum_{\boldsymbol{j}} b_{\boldsymbol{j}}^{\dagger} b_{\boldsymbol{j}}
$$

We can rewrite the above quadratic terms by using $a_{\boldsymbol{i}}=\sqrt{\frac{2}{N}} \sum_{\boldsymbol{q}} e^{i \boldsymbol{q} \cdot \boldsymbol{i}} a_{\boldsymbol{q}}, b_{\boldsymbol{j}}^{\dagger}=\sqrt{\frac{2}{N}} \sum_{\boldsymbol{q}} e^{i \boldsymbol{q} \cdot \boldsymbol{j}} b_{\boldsymbol{q}}^{\dagger}$, and $J(\boldsymbol{q})=J \sum_{\boldsymbol{\delta}} e^{i \boldsymbol{q} \cdot \boldsymbol{\delta}}$. As a result, we obtain

$$
H_{\mathrm{KE}}=\{2 S[J(\mathbf{0})+K]+h\} \sum_{\boldsymbol{q}} a_{\boldsymbol{q}}^{\dagger} a_{\boldsymbol{q}}+\{2 S[J(\mathbf{0})+K]-h\} \sum_{\boldsymbol{q}} b_{\boldsymbol{q}}^{\dagger} b_{\boldsymbol{q}}+2 S \sum_{\boldsymbol{q}} J(\boldsymbol{q})\left(a_{\boldsymbol{q}} b_{\boldsymbol{q}}+a_{\boldsymbol{q}}^{\dagger} b_{\boldsymbol{q}}^{\dagger}\right) .
$$

This is Eq. (4). Then the quartic terms which arise from the Heisenberg interaction are given by

$$
H_{\mathrm{int}}=-2 J \sum_{\langle\boldsymbol{i}, \boldsymbol{j}\rangle} a_{\boldsymbol{i}}^{\dagger} a_{\boldsymbol{i}} b_{\boldsymbol{j}}^{\dagger} b_{\boldsymbol{j}}-\frac{J}{2} \sum_{\langle\boldsymbol{i}, \boldsymbol{j}\rangle}\left(a_{\boldsymbol{i}} b_{\boldsymbol{j}}^{\dagger} b_{\boldsymbol{j}} b_{\boldsymbol{j}}+a_{\boldsymbol{i}}^{\dagger} a_{\boldsymbol{i}} a_{\boldsymbol{i}} b_{\boldsymbol{j}}+a_{\boldsymbol{i}}^{\dagger} b_{\boldsymbol{j}}^{\dagger} b_{\boldsymbol{j}}^{\dagger} b_{\boldsymbol{j}}+a_{\boldsymbol{i}}^{\dagger} a_{\boldsymbol{i}}^{\dagger} a_{\boldsymbol{i}} \boldsymbol{b}_{\boldsymbol{j}}^{\dagger}\right) \text {. }
$$


The above terms can be divided into two parts: $H_{\text {int }}=V+V^{\dagger}$, where

$$
V=-J \sum_{\langle i, \boldsymbol{j}\rangle} a_{i}^{\dagger} a_{i} b_{j}^{\dagger} b_{j}-\frac{J}{2} \sum_{\langle i, \boldsymbol{j}\rangle}\left(a_{i} b_{j}^{\dagger} b_{j} b_{j}+a_{i}^{\dagger} a_{i} a_{i} b_{j}\right)
$$

By using $a_{\boldsymbol{i}}=\sqrt{\frac{2}{N}} \sum_{\boldsymbol{q}} e^{i \boldsymbol{q} \cdot \boldsymbol{i}} a_{\boldsymbol{q}}, b_{\boldsymbol{j}}^{\dagger}=\sqrt{\frac{2}{N}} \sum_{\boldsymbol{q}} e^{i \boldsymbol{q} \cdot \boldsymbol{j}} b_{\boldsymbol{q}}^{\dagger}$, and $J(\boldsymbol{q})=J \sum_{\boldsymbol{\delta}} e^{i \boldsymbol{q} \cdot \boldsymbol{\delta}}$, we can write Eq. A4 as

$$
V=-\frac{1}{N} \sum_{\boldsymbol{q}_{1}, \boldsymbol{q}_{2}, \boldsymbol{q}_{3}, \boldsymbol{q}_{4}}\left[2 J\left(\boldsymbol{q}_{1}-\boldsymbol{q}_{3}\right) a_{\boldsymbol{q}_{1}}^{\dagger} a_{\boldsymbol{q}_{3}} b_{\boldsymbol{q}_{4}}^{\dagger} b_{\boldsymbol{q}_{2}}+J\left(\boldsymbol{q}_{1}\right)\left(a_{\boldsymbol{q}_{1}} b_{\boldsymbol{q}_{2}}^{\dagger} b_{\boldsymbol{q}_{3}} b_{\boldsymbol{q}_{4}}+a_{\boldsymbol{q}_{2}}^{\dagger} a_{\boldsymbol{q}_{3}} a_{\boldsymbol{q}_{4}} b_{\boldsymbol{q}_{1}}\right)\right] \delta_{\boldsymbol{q}_{1}+\boldsymbol{q}_{2}, \boldsymbol{q}_{3}+\boldsymbol{q}_{4}} .
$$

Since the dominant terms of $V$ come from the diagonal terms $\frac{1113 \mid 19}{}$, the terms for $\boldsymbol{q}_{1}=\boldsymbol{q}_{3}=\boldsymbol{q}, \boldsymbol{q}_{2}=\boldsymbol{q}_{4}=\boldsymbol{q}^{\prime}$ and for $\boldsymbol{q}_{1}=\boldsymbol{q}_{4}=\boldsymbol{q}, \boldsymbol{q}_{2}=\boldsymbol{q}_{3}=\boldsymbol{q}^{\prime}$, the dominant terms of $V$ are given by

$$
V=-\frac{2}{N} \sum_{\boldsymbol{q}, \boldsymbol{q}^{\prime}}\left[J(\mathbf{0}) a_{\boldsymbol{q}}^{\dagger} a_{\boldsymbol{q}} b_{\boldsymbol{q}^{\prime}}^{\dagger} b_{\boldsymbol{q}^{\prime}}+J\left(\boldsymbol{q}-\boldsymbol{q}^{\prime}\right) a_{\boldsymbol{q}}^{\dagger} a_{\boldsymbol{q}^{\prime}} b_{\boldsymbol{q}}^{\dagger} b_{\boldsymbol{q}^{\prime}}+J(\boldsymbol{q}) a_{\boldsymbol{q}} b_{\boldsymbol{q}^{\prime}}^{\dagger} b_{\boldsymbol{q}} b_{\boldsymbol{q}^{\prime}}+J(\boldsymbol{q}) a_{\boldsymbol{q}^{\prime}}^{\dagger} a_{\boldsymbol{q}} a_{\boldsymbol{q}^{\prime}} b_{\boldsymbol{q}}\right]
$$

Combining this equation with $H_{\text {int }}=V+V^{\dagger}$, we obtain Eq. (5).

\section{Appendix B: $H_{\text {intra }}$ and $H_{\text {inter }}$ expressed in terms of the operators of the $\alpha$ - and $\beta$-band magnons}

We rewrite $H_{\text {int }}$ as the sum of $H_{\text {intra }}$ and $H_{\text {inter }}$ by using Eq. (6). Since $H_{\text {int }}$ consists of the terms quartic in the operators of $A$ - and $B$-sublattice magnons, the terms of $H_{\text {int }}$ expressed in terms of the operators of the $\alpha$ - and $\beta$-band magnons can be divided into five parts: products of four operators of the $\alpha$-band magnons, products of four operators of the $\beta$-band magnons, products of two operators of the $\alpha$-band magnons and two operators of the $\beta$-band magnons, products of three operators of the $\alpha$-band magnons and one operator of the $\beta$-band magnons, and products of one operator of the $\alpha$-band magnons and three operators of the $\beta$-band magnons. The first two parts, $H_{\alpha \alpha \alpha \alpha}$ and $H_{\beta \beta \beta \beta}$, lead to the intraband interaction, $H_{\text {intra }}=H_{\alpha \alpha \alpha \alpha}+H_{\beta \beta \beta \beta}$, whereas the latter three parts, $H_{\alpha \alpha \beta \beta}, H_{\alpha \alpha \alpha \beta}$, and $H_{\alpha \beta \beta \beta}$, lead to the interband interaction, $H_{\text {inter }}=H_{\alpha \alpha \beta \beta}+H_{\alpha \alpha \alpha \beta}+H_{\alpha \beta \beta \beta}$. By expressing $H_{\text {int }}$ in terms of the operators of the $\alpha$ - and $\beta$-band magnons, we obtain $H_{\alpha \alpha \alpha \alpha}=V_{1}+V_{1}^{\dagger}, H_{\beta \beta \beta \beta}=V_{2}+V_{2}^{\dagger}, H_{\alpha \alpha \beta \beta}=V_{3}+V_{3}^{\dagger}$, $H_{\alpha \alpha \alpha \beta}=V_{4}+V_{4}^{\dagger}$, and $H_{\alpha \beta \beta \beta}=V_{5}+V_{5}^{\dagger}$, where

$$
\begin{aligned}
V_{1}= & -\frac{2}{N} \sum_{\boldsymbol{q}, \boldsymbol{q}^{\prime}} J(\mathbf{0}) c_{\boldsymbol{q}}^{2} s_{\boldsymbol{q}^{\prime}}^{2} \alpha_{\boldsymbol{q}^{\prime}}^{\dagger} \alpha_{\boldsymbol{q}} \alpha_{\boldsymbol{q}^{\prime}} \alpha_{\boldsymbol{q}^{\prime}}^{\dagger}-\frac{2}{N} \sum_{\boldsymbol{q}, \boldsymbol{q}^{\prime}} J\left(\boldsymbol{q}-\boldsymbol{q}^{\prime}\right) c_{\boldsymbol{q}} s_{\boldsymbol{q}} c_{\boldsymbol{q}^{\prime}} s_{\boldsymbol{q}^{\prime}} \alpha_{\boldsymbol{q}^{\prime}}^{\dagger} \alpha_{\boldsymbol{q}^{\prime}} \alpha_{\boldsymbol{q}} \alpha_{\boldsymbol{q}^{\prime}}^{\dagger}+\frac{2}{N} \sum_{\boldsymbol{q}, \boldsymbol{q}^{\prime}} J(\boldsymbol{q}) c_{\boldsymbol{q}} s_{\boldsymbol{q}} s_{\boldsymbol{q}^{\prime}}^{2} \alpha_{\boldsymbol{q}} \alpha_{\boldsymbol{q}^{\prime}} \alpha_{\boldsymbol{q}}^{\dagger} \alpha_{\boldsymbol{q}^{\prime}}^{\dagger} \\
& +\frac{2}{N} \sum_{\boldsymbol{q}, \boldsymbol{q}^{\prime}} J(\boldsymbol{q}) c_{\boldsymbol{q}} s_{\boldsymbol{q}} c_{\boldsymbol{q}^{\prime}}^{2} \alpha_{\boldsymbol{q}^{\prime}}^{\dagger} \alpha_{\boldsymbol{q}} \alpha_{\boldsymbol{q}^{\prime}} \alpha_{\boldsymbol{q}}^{\dagger} \\
V_{2}= & -\frac{2}{N} \sum_{\boldsymbol{q}, \boldsymbol{q}^{\prime}} J(\mathbf{0}) c_{\boldsymbol{q}^{\prime}}^{2} s_{\boldsymbol{q}}^{2} \beta_{\boldsymbol{q}} \beta_{\boldsymbol{q}}^{\dagger} \beta_{\boldsymbol{q}^{\prime}}^{\dagger} \beta_{\boldsymbol{q}^{\prime}}-\frac{2}{N} \sum_{\boldsymbol{q}, \boldsymbol{q}^{\prime}} J\left(\boldsymbol{q}-\boldsymbol{q}^{\prime}\right) c_{\boldsymbol{q}} s_{\boldsymbol{q}} c_{\boldsymbol{q}^{\prime}} s_{\boldsymbol{q}^{\prime}} \beta_{\boldsymbol{q}} \beta_{\boldsymbol{q}^{\prime}}^{\dagger} \beta_{\boldsymbol{q}^{\prime}}^{\dagger} \beta_{\boldsymbol{q}^{\prime}}+\frac{2}{N} \sum_{\boldsymbol{q}, \boldsymbol{q}^{\prime}} J(\boldsymbol{q}) c_{\boldsymbol{q}} s_{\boldsymbol{q}} c_{\boldsymbol{q}^{\prime}}^{2} \beta_{\boldsymbol{q}^{\prime}}^{\dagger} \beta_{\boldsymbol{q}^{\prime}}^{\dagger} \beta_{\boldsymbol{q}^{\prime}} \beta_{\boldsymbol{q}^{\prime}} \\
& +\frac{2}{N} \sum_{\boldsymbol{q}, \boldsymbol{q}^{\prime}} J(\boldsymbol{q}) c_{\boldsymbol{q}} s_{\boldsymbol{q}} s_{\boldsymbol{q}^{\prime}}^{2} \beta_{\boldsymbol{q}^{\prime}} \beta_{\boldsymbol{q}}^{\dagger} \beta_{\boldsymbol{q}^{\prime}}^{\dagger} \beta_{\boldsymbol{q}}
\end{aligned}
$$




$$
\begin{aligned}
& V_{3}=-\frac{2}{N} \sum_{\boldsymbol{q}, \boldsymbol{q}^{\prime}} J(\mathbf{0})\left(c_{\boldsymbol{q}}^{2} c_{\boldsymbol{q}^{\prime}}^{2} \alpha_{\boldsymbol{q}}^{\dagger} \alpha_{\boldsymbol{q}} \beta_{\boldsymbol{q}^{\prime}}^{\dagger} \beta_{\boldsymbol{q}^{\prime}}+c_{\boldsymbol{q}} s_{\boldsymbol{q}} c_{\boldsymbol{q}^{\prime}} s_{\boldsymbol{q}^{\prime}} \alpha_{\boldsymbol{q}}^{\dagger} \beta_{\boldsymbol{q}}^{\dagger} \alpha_{\boldsymbol{q}^{\prime}} \beta_{\boldsymbol{q}^{\prime}}+c_{\boldsymbol{q}} s_{\boldsymbol{q}} c_{\boldsymbol{q}^{\prime}} s_{\boldsymbol{q}^{\prime}} \alpha_{\boldsymbol{q}}^{\dagger} \beta_{\boldsymbol{q}}^{\dagger} \beta_{\boldsymbol{q}^{\prime}}^{\dagger} \alpha_{\boldsymbol{q}^{\prime}}^{\dagger}\right. \\
& \left.+c_{\boldsymbol{q}} s_{\boldsymbol{q}} c_{\boldsymbol{q}^{\prime}} s_{\boldsymbol{q}^{\prime}} \beta_{\boldsymbol{q}} \alpha_{\boldsymbol{q}} \alpha_{\boldsymbol{q}^{\prime}} \beta_{\boldsymbol{q}^{\prime}}+c_{\boldsymbol{q}} s_{\boldsymbol{q}} c_{\boldsymbol{q}^{\prime}} s_{\boldsymbol{q}^{\prime}} \beta_{\boldsymbol{q}} \alpha_{\boldsymbol{q}} \beta_{\boldsymbol{q}^{\prime}}^{\dagger} \alpha_{\boldsymbol{q}^{\prime}}^{\dagger}+s_{\boldsymbol{q}^{\prime}}^{2} s_{\boldsymbol{q}^{\prime}}^{2} \beta_{\boldsymbol{q}} \beta_{\boldsymbol{q}}^{\dagger} \alpha_{\boldsymbol{q}^{\prime}} \alpha_{\boldsymbol{q}^{\prime}}^{\dagger}\right) \\
& -\frac{2}{N} \sum_{\boldsymbol{q}, \boldsymbol{q}^{\prime}} J\left(\boldsymbol{q}-\boldsymbol{q}^{\prime}\right)\left(c_{\boldsymbol{q}}^{2} c_{\boldsymbol{q}^{\prime}}^{2} \alpha_{\boldsymbol{q}}^{\dagger} \alpha_{\boldsymbol{q}^{\prime}} \beta_{\boldsymbol{q}}^{\dagger} \beta_{\boldsymbol{q}^{\prime}}+c_{\boldsymbol{q}} s_{\boldsymbol{q}} c_{\boldsymbol{q}^{\prime}} s_{\boldsymbol{q}^{\prime}} \alpha_{\boldsymbol{q}}^{\dagger} \beta_{\boldsymbol{q}^{\prime}}^{\dagger} \alpha_{\boldsymbol{q}} \beta_{\boldsymbol{q}^{\prime}}+c_{\boldsymbol{q}} s_{\boldsymbol{q}} c_{\boldsymbol{q}^{\prime}} s_{\boldsymbol{q}^{\prime}} \beta_{\boldsymbol{q}} \alpha_{\boldsymbol{q}^{\prime}} \beta_{\boldsymbol{q}}^{\dagger} \alpha_{\boldsymbol{q}^{\prime}}^{\dagger}\right. \\
& \left.+c_{\boldsymbol{q}}^{2} s_{\boldsymbol{q}^{\prime}}^{2} \alpha_{\boldsymbol{q}}^{\dagger} \beta_{\boldsymbol{q}^{\prime}}^{\dagger} \beta_{\boldsymbol{q}}^{\dagger} \alpha_{\boldsymbol{q}^{\prime}}^{\dagger}+c_{\boldsymbol{q}^{2}}^{2} s_{\boldsymbol{q}^{\prime}}^{2} \beta_{\boldsymbol{q}^{\prime}} \alpha_{\boldsymbol{q}} \alpha_{\boldsymbol{q}^{\prime}} \beta_{\boldsymbol{q}}+s_{\boldsymbol{q}^{2}}^{2} s_{\boldsymbol{q}^{\prime}}^{2} \beta_{\boldsymbol{q}} \beta_{\boldsymbol{q}^{\prime}}^{\dagger} \alpha_{\boldsymbol{q}} \alpha_{\boldsymbol{q}^{\prime}}^{\dagger}\right) \\
& +\frac{2}{N} \sum_{\boldsymbol{q}, \boldsymbol{q}^{\prime}} J(\boldsymbol{q})\left(c_{\boldsymbol{q}}^{2} c_{\boldsymbol{q}^{\prime}} s_{\boldsymbol{q}^{\prime}} \alpha_{\boldsymbol{q}} \alpha_{\boldsymbol{q}^{\prime}} \beta_{\boldsymbol{q}} \beta_{\boldsymbol{q}^{\prime}}+c_{\boldsymbol{q}}^{2} c_{\boldsymbol{q}^{\prime}} s_{\boldsymbol{q}^{\prime}} \alpha_{\boldsymbol{q}} \beta_{\boldsymbol{q}^{\prime}}^{\dagger} \beta_{\boldsymbol{q}^{\prime}} \alpha_{\boldsymbol{q}^{\prime}}^{\dagger}+c_{\boldsymbol{q}^{\prime}}^{2} c_{\boldsymbol{q}} s_{\boldsymbol{q}} \alpha_{\boldsymbol{q}} \beta_{\boldsymbol{q}^{\prime}}^{\dagger} \alpha_{\boldsymbol{q}}^{\dagger} \beta_{\boldsymbol{q}^{\prime}}\right. \\
& \left.+s_{\boldsymbol{q}^{\prime}}^{2} c_{\boldsymbol{q}^{\prime}} s_{\boldsymbol{q}^{\prime}} \beta_{\boldsymbol{q}^{\prime}}^{\dagger} \alpha_{\boldsymbol{q}^{\prime}} \alpha_{\boldsymbol{q}}^{\dagger} \beta_{\boldsymbol{q}^{\prime}}+s_{\boldsymbol{q}}^{2} c_{\boldsymbol{q}^{\prime}} s_{\boldsymbol{q}^{\prime}} \beta_{\boldsymbol{q}}^{\dagger} \beta_{\boldsymbol{q}^{\prime}}^{\dagger} \alpha_{\boldsymbol{q}}^{\dagger} \alpha_{\boldsymbol{q}^{\prime}}^{\dagger}+s_{\boldsymbol{q}^{\prime}}^{2} c_{\boldsymbol{q}} s_{\boldsymbol{q}} \beta_{\boldsymbol{q}}^{\dagger} \alpha_{\boldsymbol{q}^{\prime}} \beta_{\boldsymbol{q}} \alpha_{\boldsymbol{q}^{\prime}}^{\dagger}\right) \\
& +\frac{2}{N} \sum_{\boldsymbol{q}, \boldsymbol{q}^{\prime}} J(\boldsymbol{q})\left(c_{\boldsymbol{q}}^{2} c_{\boldsymbol{q}^{\prime}} s_{\boldsymbol{q}^{\prime}} \alpha_{\boldsymbol{q}^{\prime}}^{\dagger} \alpha_{\boldsymbol{q}} \beta_{\boldsymbol{q}^{\prime}}^{\dagger} \beta_{\boldsymbol{q}}+c_{\boldsymbol{q}}^{2} c_{\boldsymbol{q}^{\prime}} s_{\boldsymbol{q}^{\prime}} \beta_{\boldsymbol{q}^{\prime}} \alpha_{\boldsymbol{q}} \alpha_{\boldsymbol{q}^{\prime}} \beta_{\boldsymbol{q}}+c_{\boldsymbol{q}^{\prime}}^{2} c_{\boldsymbol{q}} s_{\boldsymbol{q}} \alpha_{\boldsymbol{q}^{\prime}}^{\dagger} \beta_{\boldsymbol{q}^{\prime}}^{\dagger} \alpha_{\boldsymbol{q}^{\prime}} \beta_{\boldsymbol{q}}\right. \\
& \left.+s_{\boldsymbol{q}}^{2} c_{\boldsymbol{q}^{\prime}} s_{\boldsymbol{q}^{\prime}} \alpha_{\boldsymbol{q}^{\prime}}^{\dagger} \beta_{\boldsymbol{q}}^{\dagger} \beta_{\boldsymbol{q}^{\prime}}^{\dagger} \alpha_{\boldsymbol{q}}^{\dagger}+s_{\boldsymbol{q}}^{2} c_{\boldsymbol{q}^{\prime}} s_{\boldsymbol{q}^{\prime}} \beta_{\boldsymbol{q}^{\prime}} \beta_{\boldsymbol{q}^{\prime}}^{\dagger} \alpha_{\boldsymbol{q}^{\prime}} \alpha_{\boldsymbol{q}}^{\dagger}+s_{\boldsymbol{q}^{\prime}}^{2} c_{\boldsymbol{q}} s_{\boldsymbol{q}} \beta_{\boldsymbol{q}^{\prime}} \alpha_{\boldsymbol{q}} \beta_{\boldsymbol{q}^{\prime}}^{\dagger} \alpha_{\boldsymbol{q}}^{\dagger}\right), \\
& V_{4}=\frac{2}{N} \sum_{\boldsymbol{q}, \boldsymbol{q}^{\prime}} J(\mathbf{0})\left(s_{\boldsymbol{q}^{\prime}}^{2} c_{\boldsymbol{q}} s_{\boldsymbol{q}} \beta_{\boldsymbol{q}} \alpha_{\boldsymbol{q}} \alpha_{\boldsymbol{q}^{\prime}} \alpha_{\boldsymbol{q}^{\prime}}^{\dagger}+s_{\boldsymbol{q}^{\prime}}^{2} c_{\boldsymbol{q}} s_{\boldsymbol{q}} \alpha_{\boldsymbol{q}}^{\dagger} \beta_{\boldsymbol{q}}^{\dagger} \alpha_{\boldsymbol{q}^{\prime}} \alpha_{\boldsymbol{q}^{\prime}}^{\dagger}+c_{\boldsymbol{q}}^{2} c_{\boldsymbol{q}^{\prime}} s_{\boldsymbol{q}^{\prime}} \alpha_{\boldsymbol{q}}^{\dagger} \alpha_{\boldsymbol{q}} \beta_{\boldsymbol{q}^{\prime}}^{\dagger} \alpha_{\boldsymbol{q}^{\prime}}^{\dagger}+c_{\boldsymbol{q}}^{2} c_{\boldsymbol{q}^{\prime}} s_{\boldsymbol{q}^{\prime}} \alpha_{\boldsymbol{q}^{\prime}}^{\dagger} \alpha_{\boldsymbol{q}} \alpha_{\boldsymbol{q}^{\prime}} \beta_{\boldsymbol{q}^{\prime}}\right) \\
& +\frac{2}{N} \sum_{\boldsymbol{q}, \boldsymbol{q}^{\prime}} J\left(\boldsymbol{q}-\boldsymbol{q}^{\prime}\right)\left(s_{\boldsymbol{q}^{\prime}}^{2} c_{\boldsymbol{q}} s_{\boldsymbol{q}} \beta_{\boldsymbol{q}^{\prime}} \alpha_{\boldsymbol{q}} \alpha_{\boldsymbol{q}^{\prime}} \alpha_{\boldsymbol{q}}^{\dagger}+s_{\boldsymbol{q}^{\prime}}^{2} c_{\boldsymbol{q}} s_{\boldsymbol{q}} \alpha_{\boldsymbol{q}}^{\dagger} \beta_{\boldsymbol{q}^{\prime}}^{\dagger} \alpha_{\boldsymbol{q}} \alpha_{\boldsymbol{q}^{\prime}}^{\dagger}+c_{\boldsymbol{q}}^{2} c_{\boldsymbol{q}^{\prime}} s_{\boldsymbol{q}^{\prime}} \alpha_{\boldsymbol{q}}^{\dagger} \alpha_{\boldsymbol{q}^{\prime}} \beta_{\boldsymbol{q}^{\prime}}^{\dagger} \alpha_{\boldsymbol{q}^{\prime}}^{\dagger}+c_{\boldsymbol{q}^{\prime}}^{2} c_{\boldsymbol{q}^{\prime}} s_{\boldsymbol{q}^{\prime}} \alpha_{\boldsymbol{q}^{\prime}}^{\dagger} \alpha_{\boldsymbol{q}} \alpha_{\boldsymbol{q}^{\prime}} \beta_{\boldsymbol{q}}\right) \\
& -\frac{2}{N} \sum_{\boldsymbol{q}, \boldsymbol{q}^{\prime}} J(\boldsymbol{q})\left(s_{\boldsymbol{q}}^{2} s_{\boldsymbol{q}^{\prime}}^{2} \beta_{\boldsymbol{q}}^{\dagger} \alpha_{\boldsymbol{q}^{\prime}} \alpha_{\boldsymbol{q}}^{\dagger} \alpha_{\boldsymbol{q}^{\prime}}^{\dagger}+c_{\boldsymbol{q}} s_{\boldsymbol{q}} c_{\boldsymbol{q}^{\prime}} s_{\boldsymbol{q}^{\prime}} \alpha_{\boldsymbol{q}} \beta_{\boldsymbol{q}^{\prime}}^{\dagger} \alpha_{\boldsymbol{q}}^{\dagger} \alpha_{\boldsymbol{q}^{\prime}}^{\dagger}+c_{\boldsymbol{q}} s_{\boldsymbol{q}} c_{\boldsymbol{q}^{\prime}} s_{\boldsymbol{q}^{\prime}} \alpha_{\boldsymbol{q}} \alpha_{\boldsymbol{q}^{\prime}} \alpha_{\boldsymbol{q}}^{\dagger} \beta_{\boldsymbol{q}^{\prime}}+c_{\boldsymbol{q}}^{2} s_{\boldsymbol{q}^{\prime}}^{2} \alpha_{\boldsymbol{q}} \alpha_{\boldsymbol{q}^{\prime}} \beta_{\boldsymbol{q}} \alpha_{\boldsymbol{q}^{\prime}}^{\dagger}\right) \\
& -\frac{2}{N} \sum_{\boldsymbol{q}, \boldsymbol{q}^{\prime}} J(\boldsymbol{q})\left(c_{\boldsymbol{q}} s_{\boldsymbol{q}} c_{\boldsymbol{q}^{\prime}} s_{\boldsymbol{q}^{\prime}} \beta_{\boldsymbol{q}^{\prime}} \alpha_{\boldsymbol{q}} \alpha_{\boldsymbol{q}^{\prime}} \alpha_{\boldsymbol{q}}^{\dagger}+c_{\boldsymbol{q}} s_{\boldsymbol{q}} c_{\boldsymbol{q}^{\prime}} s_{\boldsymbol{q}^{\prime}} \alpha_{\boldsymbol{q}^{\prime}}^{\dagger} \alpha_{\boldsymbol{q}} \beta_{\boldsymbol{q}^{\prime}}^{\dagger} \alpha_{\boldsymbol{q}}^{\dagger}+s_{\boldsymbol{q}}^{2} c_{\boldsymbol{q}^{\prime}}^{2} \alpha_{\boldsymbol{q}^{\prime}}^{\dagger} \beta_{\boldsymbol{q}}^{\dagger} \alpha_{\boldsymbol{q}^{\prime}} \alpha_{\boldsymbol{q}}^{\dagger}+c_{\boldsymbol{q}}^{2} c_{\boldsymbol{q}^{\prime}}^{2} \alpha_{\boldsymbol{q}^{\prime}}^{\dagger} \alpha_{\boldsymbol{q}} \alpha_{\boldsymbol{q}^{\prime}} \beta_{\boldsymbol{q}}\right),
\end{aligned}
$$

and

$$
\begin{aligned}
& V_{5}=\frac{2}{N} \sum_{\boldsymbol{q}, \boldsymbol{q}^{\prime}} J(\mathbf{0})\left(c_{\boldsymbol{q}^{\prime}}^{2} c_{\boldsymbol{q}} s_{\boldsymbol{q}} \alpha_{\boldsymbol{q}}^{\dagger} \beta_{\boldsymbol{q}}^{\dagger} \beta_{\boldsymbol{q}^{\prime}}^{\dagger} \beta_{\boldsymbol{q}^{\prime}}+c_{\boldsymbol{q}^{\prime}}^{2} c_{\boldsymbol{q}} s_{\boldsymbol{q}} \beta_{\boldsymbol{q}} \alpha_{\boldsymbol{q}} \beta_{\boldsymbol{q}^{\prime}}^{\dagger} \beta_{\boldsymbol{q}^{\prime}}+s_{\boldsymbol{q}^{\prime}}^{2} c_{\boldsymbol{q}^{\prime}} s_{\boldsymbol{q}^{\prime}} \beta_{\boldsymbol{q}} \beta_{\boldsymbol{q}}^{\dagger} \alpha_{\boldsymbol{q}^{\prime}} \beta_{\boldsymbol{q}^{\prime}}+s_{\boldsymbol{q}}^{2} c_{\boldsymbol{q}^{\prime}} s_{\boldsymbol{q}^{\prime}} \beta_{\boldsymbol{q}} \beta_{\boldsymbol{q}}^{\dagger} \beta_{\boldsymbol{q}^{\prime}}^{\dagger} \alpha_{\boldsymbol{q}^{\prime}}^{\dagger}\right) \\
& +\frac{2}{N} \sum_{\boldsymbol{q}, \boldsymbol{q}^{\prime}} J\left(\boldsymbol{q}-\boldsymbol{q}^{\prime}\right)\left(c_{\boldsymbol{q}}^{2} c_{\boldsymbol{q}^{\prime}} s_{\boldsymbol{q}^{\prime}} \alpha_{\boldsymbol{q}}^{\dagger} \beta_{\boldsymbol{q}^{\prime}}^{\dagger} \beta_{\boldsymbol{q}}^{\dagger} \beta_{\boldsymbol{q}^{\prime}}+c_{\boldsymbol{q}^{\prime}}^{2} c_{\boldsymbol{q}} s_{\boldsymbol{q}} \beta_{\boldsymbol{q}} \alpha_{\boldsymbol{q}^{\prime}} \beta_{\boldsymbol{q}}^{\dagger} \beta_{\boldsymbol{q}^{\prime}}+s_{\boldsymbol{q}}^{2} c_{\boldsymbol{q}^{\prime}} s_{\boldsymbol{q}^{\prime}} \beta_{\boldsymbol{q}} \beta_{\boldsymbol{q}^{\prime}}^{\dagger} \alpha_{\boldsymbol{q}} \beta_{\boldsymbol{q}^{\prime}}+s_{\boldsymbol{q}^{\prime}}^{2} c_{\boldsymbol{q}} s_{\boldsymbol{q}} \beta_{\boldsymbol{q}} \beta_{\boldsymbol{q}^{\prime}}^{\dagger} \beta_{\boldsymbol{q}^{\prime}}^{\dagger} \alpha_{\boldsymbol{q}^{\prime}}^{\dagger}\right) \\
& -\frac{2}{N} \sum_{\boldsymbol{q}, \boldsymbol{q}^{\prime}} J(\boldsymbol{q})\left(c_{\boldsymbol{q}}^{2} c_{\boldsymbol{q}^{\prime}}^{2} \alpha_{\boldsymbol{q}} \beta_{\boldsymbol{q}^{\prime}}^{\dagger} \beta_{\boldsymbol{q}} \beta_{\boldsymbol{q}^{\prime}}+c_{\boldsymbol{q}} s_{\boldsymbol{q}} c_{\boldsymbol{q}^{\prime}} s_{\boldsymbol{q}^{\prime}} \beta_{\boldsymbol{q}}^{\dagger} \alpha_{\boldsymbol{q}^{\prime}} \beta_{\boldsymbol{q}} \beta_{\boldsymbol{q}^{\prime}}+c_{\boldsymbol{q}} s_{\boldsymbol{q}} c_{\boldsymbol{q}^{\prime}} s_{\boldsymbol{q}^{\prime}} \beta_{\boldsymbol{q}}^{\dagger} \beta_{\boldsymbol{q}^{\prime}}^{\dagger} \beta_{\boldsymbol{q}} \alpha_{\boldsymbol{q}^{\prime}}^{\dagger}+s_{\boldsymbol{q}}^{2} c_{\boldsymbol{q}^{\prime}}^{2} \beta_{\boldsymbol{q}^{\prime}}^{\dagger} \beta_{\boldsymbol{q}^{\prime}}^{\dagger} \alpha_{\boldsymbol{q}}^{\dagger} \beta_{\boldsymbol{q}^{\prime}}\right) \\
& -\frac{2}{N} \sum_{\boldsymbol{q}, \boldsymbol{q}^{\prime}} J(\boldsymbol{q})\left(c_{\boldsymbol{q}} s_{\boldsymbol{q}} c_{\boldsymbol{q}^{\prime}} s_{\boldsymbol{q}^{\prime}} \alpha_{\boldsymbol{q}^{\prime}}^{\dagger} \beta_{\boldsymbol{q}}^{\dagger} \beta_{\boldsymbol{q}^{\prime}}^{\dagger} \beta_{\boldsymbol{q}}+c_{\boldsymbol{q}}^{2} s_{\boldsymbol{q}^{\prime}}^{2} \beta_{\boldsymbol{q}^{\prime}} \alpha_{\boldsymbol{q}} \beta_{\boldsymbol{q}^{\prime}}^{\dagger} \beta_{\boldsymbol{q}}+c_{\boldsymbol{q}} s_{\boldsymbol{q}} c_{\boldsymbol{q}^{\prime}} s_{\boldsymbol{q}^{\prime}} \beta_{\boldsymbol{q}^{\prime}} \beta_{\boldsymbol{q}}^{\dagger} \alpha_{\boldsymbol{q}^{\prime}} \beta_{\boldsymbol{q}}+s_{\boldsymbol{q}^{2}}^{2} s_{\boldsymbol{q}^{\prime}}^{2} \beta_{\boldsymbol{q}^{\prime}} \beta_{\boldsymbol{q}}^{\dagger} \beta_{\boldsymbol{q}^{\prime}}^{\dagger} \alpha_{\boldsymbol{q}}^{\dagger}\right) .
\end{aligned}
$$

Therefore $H_{\text {intra }}$ is given by $H_{\text {intra }}=V_{1}+V_{2}+$ (H.c.) with Eqs. (B1) and $(\bar{B} 2)$, and $H_{\text {inter }}$ is given by $H_{\text {inter }}=$ $V_{3}+V_{4}+V_{5}+$ (H.c.) with Eqs. (B3)-(B5). Note that each term in Eqs. (B1)-(B5) consists of the terms for $\boldsymbol{q}=\boldsymbol{q}^{\prime}=\mathbf{0}$, for $\boldsymbol{q}=\mathbf{0}, \boldsymbol{q}^{\prime} \neq \mathbf{0}$, for $\boldsymbol{q} \neq \mathbf{0}, \boldsymbol{q}^{\prime}=\mathbf{0}$, and for $\boldsymbol{q} \neq \mathbf{0}, \boldsymbol{q}^{\prime} \neq \mathbf{0}$.

\section{Appendix C: Derivation of the mean-field interaction terms of $H_{\text {intra }}$ and $H_{\text {inter }}$ for $\boldsymbol{q}=\boldsymbol{q}^{\prime}=\mathbf{0}$, for $\boldsymbol{q}=\mathbf{0}$,} $q^{\prime} \neq 0$, and for $q \neq 0, q^{\prime}=0$ and leading-order estimate

We derive the mean-field interaction terms which arise from the terms of $H_{\text {int }}=H_{\text {intra }}+H_{\text {inter }}$ for $\boldsymbol{q}=\boldsymbol{q}^{\prime}=\mathbf{0}$, for $\boldsymbol{q}=\mathbf{0}, \boldsymbol{q}^{\prime} \neq \mathbf{0}$, and for $\boldsymbol{q} \neq \mathbf{0}, \boldsymbol{q}^{\prime}=\mathbf{0}$, and we estimate the coefficients within the leading order. This derivation can be performed in a manner similar to that of the Bogoliubov theory ${ }^{2425}$ for the BEC in a Bose gas. Since the condensed states in the BEC of magnons in the antiferromagnet without the external magnetic field are the $\boldsymbol{q}=\mathbf{0}$ states of the $\alpha$ - and $\beta$-band magnons, we can use the following Bogoliubov approximations: $\alpha_{\mathbf{0}} \approx \sqrt{n_{\mathbf{0} \alpha}}, \alpha_{\mathbf{0}}^{\dagger} \approx \sqrt{n_{\mathbf{0} \alpha}}, \beta_{\mathbf{0}} \approx \sqrt{n_{\mathbf{0} \beta}}$, and $\beta_{\mathbf{0}}^{\dagger} \approx \sqrt{n_{\mathbf{0} \beta}}$. Note that $\sqrt{n_{\mathbf{0} \alpha}}$ and $\sqrt{n_{\mathbf{0} \beta}}$ satisfy $\sqrt{n_{\mathbf{0} \alpha}}=\sqrt{N_{\alpha}-\sum_{\boldsymbol{q} \neq \mathbf{0}} \alpha_{\boldsymbol{q}}^{\dagger} \alpha_{\boldsymbol{q}}}$ and $\sqrt{n_{\mathbf{0} \beta}}=\sqrt{N_{\beta}-\sum_{\boldsymbol{q} \neq \mathbf{0}} \beta_{\boldsymbol{q}}^{\dagger} \beta_{\boldsymbol{q}}}$, where $N_{\alpha}$ and $N_{\beta}$ are the numbers of the $\alpha$ - and $\beta$-band magnons $\left(N_{\alpha}=N_{\beta} \equiv N_{\mathrm{m}}\right.$ is satisfied in the absence of the external magnetic field). Because of the Bogoliubov approximations, for example the first term of $V_{1}$ in Eq. (B1) 
gives the following mean-field interaction terms:

$$
-\frac{2}{N} J(\mathbf{0}) c_{\mathbf{0}}^{2} s_{\mathbf{0}}^{2} n_{\mathbf{0} \alpha}^{2}-\frac{2}{N} \sum_{\boldsymbol{q}^{\prime} \neq \mathbf{0}} J(\mathbf{0}) c_{\mathbf{0}}^{2} s_{\boldsymbol{q}^{\prime}}^{2} n_{\mathbf{0} \alpha} \alpha_{\boldsymbol{q}^{\prime}} \alpha_{\boldsymbol{q}^{\prime}}^{\dagger}-\frac{2}{N} \sum_{\boldsymbol{q} \neq \mathbf{0}} J(\mathbf{0}) c_{\boldsymbol{q}}^{2} s_{\mathbf{0}}^{2} n_{\mathbf{0} \alpha} \alpha_{\boldsymbol{q}}^{\dagger} \alpha_{\boldsymbol{q}}
$$

(Here we have neglected the contribution for $\boldsymbol{q} \neq \mathbf{0}, \boldsymbol{q}^{\prime} \neq \mathbf{0}$.) In a similar manner we can derive the mean-field interaction terms for $\boldsymbol{q}=\boldsymbol{q}^{\prime}=\mathbf{0}$, for $\boldsymbol{q}=\mathbf{0}, \boldsymbol{q}^{\prime} \neq \mathbf{0}$, and for $\boldsymbol{q} \neq \mathbf{0}, \boldsymbol{q}^{\prime}=\mathbf{0}$ from the other terms of $V_{1}$ and the terms of $V_{2}, V_{3}, V_{4}$, and $V_{5}$. By performing a similar calculation for each term in Eqs. (B1) - B5) and combining these results with $H_{\alpha \alpha \alpha \alpha}=V_{1}+V_{1}^{\dagger}, H_{\beta \beta \beta \beta}=V_{2}+V_{2}^{\dagger}, H_{\alpha \alpha \beta \beta}=V_{3}+V_{3}^{\dagger}, H_{\alpha \alpha \alpha \beta}=V_{4}+V_{4}^{\dagger}$, and $H_{\alpha \beta \beta \beta}=V_{5}+V_{5}^{\dagger}$, we can write the mean-field interaction terms which arise from the terms for $\boldsymbol{q}=\boldsymbol{q}^{\prime}=\mathbf{0}$, for $\boldsymbol{q}=\mathbf{0}, \boldsymbol{q}^{\prime} \neq \mathbf{0}$, and for $\boldsymbol{q} \neq \mathbf{0}, \boldsymbol{q}^{\prime}=\mathbf{0}$ as $H_{\alpha \alpha \alpha \alpha}^{(0)}, H_{\beta \beta \beta \beta}^{(0)}, H_{\alpha \alpha \beta \beta}^{(0)}, H_{\alpha \alpha \alpha \beta}^{(0)}$, and $H_{\alpha \beta \beta \beta}^{(0)}$, where

$$
\begin{aligned}
& H_{\alpha \alpha \alpha \alpha}^{(0)}=\frac{4}{N} N_{\mathrm{m}}^{2} J(\mathbf{0}) c_{\mathbf{0}} s_{\mathbf{0}}\left(c_{\mathbf{0}}^{2}+s_{\mathbf{0}}^{2}-2 c_{\mathbf{0}} s_{\mathbf{0}}\right)-\frac{4}{N} N_{\mathrm{m}} J(\mathbf{0}) c_{\mathbf{0}} s_{\mathbf{0}}\left(c_{\mathbf{0}}^{2}+s_{\mathbf{0}}^{2}-2 c_{\mathbf{0}} s_{\mathbf{0}}\right) \sum_{\boldsymbol{q} \neq \mathbf{0}} \alpha_{\boldsymbol{q}}^{\dagger} \alpha_{\boldsymbol{q}} \\
& -\frac{4}{N} N_{\mathrm{m}} \sum_{\boldsymbol{q} \neq \mathbf{0}} \alpha_{\boldsymbol{q}}^{\dagger} \alpha_{\boldsymbol{q}}\left[J(\mathbf{0})\left(c_{\boldsymbol{q}}^{2} s_{\mathbf{0}}^{2}+c_{\mathbf{0}}^{2} s_{\boldsymbol{q}}^{2}\right)+2 J(\boldsymbol{q}) c_{\boldsymbol{q}} s_{\boldsymbol{q}} c_{\mathbf{0}} s_{\mathbf{0}}-J(\mathbf{0}) c_{\mathbf{0}} s_{\mathbf{0}}\left(c_{\boldsymbol{q}}^{2}+s_{\boldsymbol{q}}^{2}\right)-J(\boldsymbol{q}) c_{\boldsymbol{q}} s_{\boldsymbol{q}}\left(c_{\mathbf{0}}^{2}+s_{\mathbf{0}}^{2}\right)\right], \\
& H_{\beta \beta \beta \beta}^{(0)}=\frac{4}{N} N_{\mathrm{m}}^{2} J(\mathbf{0}) c_{\mathbf{0}} s_{\mathbf{0}}\left(c_{\mathbf{0}}^{2}+s_{\mathbf{0}}^{2}-2 c_{\mathbf{0}} s_{\mathbf{0}}\right)-\frac{4}{N} N_{\mathrm{m}} J(\mathbf{0}) c_{\mathbf{0}} s_{\mathbf{0}}\left(c_{\mathbf{0}}^{2}+s_{\mathbf{0}}^{2}-2 c_{\mathbf{0}} s_{\mathbf{0}}\right) \sum_{\boldsymbol{q} \neq \mathbf{0}} \beta_{\boldsymbol{q}}^{\dagger} \beta_{\boldsymbol{q}} \\
& -\frac{4}{N} N_{\mathrm{m}} \sum_{\boldsymbol{q} \neq \mathbf{0}} \beta_{\boldsymbol{q}}^{\dagger} \beta_{\boldsymbol{q}}\left[J(\mathbf{0})\left(c_{\boldsymbol{q}}^{2} s_{\mathbf{0}}^{2}+c_{\mathbf{0}}^{2} s_{\boldsymbol{q}}^{2}\right)+2 J(\boldsymbol{q}) c_{\boldsymbol{q}} s_{\boldsymbol{q}} c_{\mathbf{0}} s_{\mathbf{0}}-J(\mathbf{0}) c_{\mathbf{0}} s_{\mathbf{0}}\left(c_{\boldsymbol{q}}^{2}+s_{\boldsymbol{q}}^{2}\right)-J(\boldsymbol{q}) c_{\boldsymbol{q}} s_{\boldsymbol{q}}\left(c_{\mathbf{0}}^{2}+s_{\mathbf{0}}^{2}\right)\right] \\
& H_{\alpha \alpha \beta \beta}^{(0)}=-\frac{8}{N} N_{\mathrm{m}}^{2} J(\mathbf{0})\left(c_{\mathbf{0}}^{2}+s_{\mathbf{0}}^{2}-c_{\mathbf{0}} s_{\mathbf{0}}\right)\left(c_{\mathbf{0}}^{2}+s_{\mathbf{0}}^{2}-2 c_{\mathbf{0}} s_{\mathbf{0}}\right) \\
& +\frac{8}{N} N_{\mathrm{m}} J(\mathbf{0})\left(c_{\mathbf{0}}^{2}+s_{\mathbf{0}}^{2}-c_{\mathbf{0}} s_{\mathbf{0}}\right)\left(c_{\mathbf{0}}^{2}+s_{\mathbf{0}}^{2}-2 c_{\mathbf{0}} s_{\mathbf{0}}\right) \sum_{\boldsymbol{q} \neq \mathbf{0}}\left(\alpha_{\boldsymbol{q}}^{\dagger} \alpha_{\boldsymbol{q}}+\beta_{\boldsymbol{q}}^{\dagger} \beta_{\boldsymbol{q}}\right) \\
& -\frac{4}{N} N_{\mathrm{m}} \sum_{\boldsymbol{q} \neq \mathbf{0}}\left(\alpha_{\boldsymbol{q}}^{\dagger} \alpha_{\boldsymbol{q}}+\beta_{\boldsymbol{q}}^{\dagger} \beta_{\boldsymbol{q}}\right)\left\{J(\mathbf{0})\left[\left(c_{\boldsymbol{q}}^{2} c_{\mathbf{0}}^{2}+s_{\boldsymbol{q}}^{2} s_{\mathbf{0}}^{2}\right)-c_{\mathbf{0}} s_{\mathbf{0}}\left(c_{\boldsymbol{q}}^{2}+s_{\boldsymbol{q}}^{2}\right)\right]-J(\boldsymbol{q}) c_{\boldsymbol{q}} s_{\boldsymbol{q}}\left(c_{\mathbf{0}}^{2}+s_{\mathbf{0}}^{2}-2 c_{\mathbf{0}} s_{\mathbf{0}}\right)\right\} \\
& +\frac{4}{N} N_{\mathrm{m}} \sum_{\boldsymbol{q} \neq \mathbf{0}}\left(\alpha_{\boldsymbol{q}} \beta_{\boldsymbol{q}}+\alpha_{\boldsymbol{q}}^{\dagger} \beta_{\boldsymbol{q}}^{\dagger}\right)\left(c_{\mathbf{0}}^{2}+s_{\mathbf{0}}^{2}-2 c_{\mathbf{0}} s_{\mathbf{0}}\right)\left[2 J(\mathbf{0}) c_{\boldsymbol{q}} s_{\boldsymbol{q}}-J(\boldsymbol{q})\left(c_{\boldsymbol{q}}^{2}+s_{\boldsymbol{q}}^{2}\right)\right], \\
& H_{\alpha \alpha \alpha \beta}^{(0)}=-\frac{4}{N} N_{\mathrm{m}}^{2} J(\mathbf{0})+\frac{2}{N} N_{\mathrm{m}} J(\mathbf{0}) \sum_{\boldsymbol{q} \neq \mathbf{0}}\left(3 \alpha_{\boldsymbol{q}}^{\dagger} \alpha_{\boldsymbol{q}}+\beta_{\boldsymbol{q}}^{\dagger} \beta_{\boldsymbol{q}}\right) \\
& +\frac{16}{N} N_{\mathrm{m}}^{2} J(\mathbf{0}) c_{\mathbf{0}} s_{\mathbf{0}}\left(c_{\mathbf{0}}^{2}+s_{\mathbf{0}}^{2}-2 c_{\mathbf{0}} s_{\mathbf{0}}\right)-\frac{8}{N} N_{\mathrm{m}} J(\mathbf{0}) c_{\mathbf{0}} s_{\mathbf{0}}\left(c_{\mathbf{0}}^{2}+s_{\mathbf{0}}^{2}-2 c_{\mathbf{0}} s_{\mathbf{0}}\right) \sum_{\boldsymbol{q} \neq \mathbf{0}}\left(3 \alpha_{\boldsymbol{q}}^{\dagger} \alpha_{\boldsymbol{q}}+\beta_{\boldsymbol{q}}^{\dagger} \beta_{\boldsymbol{q}}\right) \\
& -\frac{4}{N} N_{\mathrm{m}}\left(c_{\mathbf{0}}^{2}+s_{\mathbf{0}}^{2}-2 c_{\mathbf{0}} s_{\mathbf{0}}\right) \sum_{\boldsymbol{q} \neq \mathbf{0}} \alpha_{\boldsymbol{q}}^{\dagger} \alpha_{\boldsymbol{q}}\left[J(\mathbf{0})\left(c_{\boldsymbol{q}}^{2}+s_{\boldsymbol{q}}^{2}\right)-2 J(\boldsymbol{q}) c_{\boldsymbol{q}} s_{\boldsymbol{q}}\right] \\
& +\frac{2}{N} N_{\mathrm{m}} \sum_{\boldsymbol{q} \neq \mathbf{0}}\left(\alpha_{\boldsymbol{q}} \beta_{\boldsymbol{q}}+\alpha_{\boldsymbol{q}}^{\dagger} \beta_{\boldsymbol{q}}^{\dagger}\right)\left(c_{\mathbf{0}}^{2}+s_{\mathbf{0}}^{2}-2 c_{\mathbf{0}} s_{\mathbf{0}}\right)\left[2 J(\mathbf{0}) c_{\boldsymbol{q}} s_{\boldsymbol{q}}-J(\boldsymbol{q})\left(c_{\boldsymbol{q}}^{2}+s_{\boldsymbol{q}}^{2}\right)\right],
\end{aligned}
$$

and

$$
\begin{aligned}
H_{\alpha \beta \beta \beta}^{(0)}= & -\frac{4}{N} N_{\mathrm{m}}^{2} J(\mathbf{0})+\frac{2}{N} N_{\mathrm{m}} J(\mathbf{0}) \sum_{\boldsymbol{q} \neq \mathbf{0}}\left(\alpha_{\boldsymbol{q}}^{\dagger} \alpha_{\boldsymbol{q}}+3 \beta_{\boldsymbol{q}}^{\dagger} \beta_{\boldsymbol{q}}\right) \\
& +\frac{16}{N} N_{\mathrm{m}}^{2} J(\mathbf{0}) c_{\mathbf{0}} s_{\mathbf{0}}\left(c_{\mathbf{0}}^{2}+s_{\mathbf{0}}^{2}-2 c_{\mathbf{0}} s_{\mathbf{0}}\right)-\frac{8}{N} N_{\mathrm{m}} J(\mathbf{0}) c_{\mathbf{0}} s_{\mathbf{0}}\left(c_{\mathbf{0}}^{2}+s_{\mathbf{0}}^{2}-2 c_{\mathbf{0}} s_{\mathbf{0}}\right) \sum_{\boldsymbol{q} \neq \mathbf{0}}\left(\alpha_{\boldsymbol{q}}^{\dagger} \alpha_{\boldsymbol{q}}+3 \beta_{\boldsymbol{q}}^{\dagger} \beta_{\boldsymbol{q}}\right) \\
& -\frac{4}{N} N_{\mathrm{m}}\left(c_{\mathbf{0}}^{2}+s_{\mathbf{0}}^{2}-2 c_{\mathbf{0}} s_{\mathbf{0}}\right) \sum_{\boldsymbol{q} \neq \mathbf{0}} \beta_{\boldsymbol{q}}^{\dagger} \beta_{\boldsymbol{q}}\left[J(\mathbf{0})\left(c_{\boldsymbol{q}}^{2}+s_{\boldsymbol{q}}^{2}\right)-2 J(\boldsymbol{q}) c_{\boldsymbol{q}} s_{\boldsymbol{q}}\right] \\
& +\frac{2}{N} N_{\mathrm{m}} \sum_{\boldsymbol{q} \neq \mathbf{0}}\left(\alpha_{\boldsymbol{q}} \beta_{\boldsymbol{q}}+\alpha_{\boldsymbol{q}}^{\dagger} \beta_{\boldsymbol{q}}^{\dagger}\right)\left(c_{\mathbf{0}}^{2}+s_{\mathbf{0}}^{2}-2 c_{\mathbf{0}} s_{\mathbf{0}}\right)\left[2 J(\mathbf{0}) c_{\boldsymbol{q}} s_{\boldsymbol{q}}-J(\boldsymbol{q})\left(c_{\boldsymbol{q}}^{2}+s_{\boldsymbol{q}}^{2}\right)\right]
\end{aligned}
$$


We have used

$$
\begin{aligned}
& n_{\mathbf{0} \alpha}^{2}=\left(N_{\alpha}-\sum_{\boldsymbol{q} \neq \mathbf{0}} \alpha_{\boldsymbol{q}}^{\dagger} \alpha_{\boldsymbol{q}}\right)^{2} \approx N_{\alpha}^{2}-2 N_{\alpha} \sum_{\boldsymbol{q} \neq \mathbf{0}} \alpha_{\boldsymbol{q}}^{\dagger} \alpha_{\boldsymbol{q}}=N_{\mathrm{m}}^{2}-2 N_{\mathrm{m}} \sum_{\boldsymbol{q} \neq \mathbf{0}} \alpha_{\boldsymbol{q}}^{\dagger} \alpha_{\boldsymbol{q}}, \\
& n_{\mathbf{0} \alpha} \sum_{\boldsymbol{q} \neq \mathbf{0}} \alpha_{\boldsymbol{q}}^{\dagger} \alpha_{\boldsymbol{q}} \approx N_{\alpha} \sum_{\boldsymbol{q} \neq \mathbf{0}} \alpha_{\boldsymbol{q}}^{\dagger} \alpha_{\boldsymbol{q}}=N_{\mathrm{m}} \sum_{\boldsymbol{q} \neq \mathbf{0}} \alpha_{\boldsymbol{q}}^{\dagger} \alpha_{\boldsymbol{q}},
\end{aligned}
$$

for the derivation of Eq. C2,

$$
\begin{aligned}
& n_{\mathbf{0} \beta}^{2}=\left(N_{\beta}-\sum_{\boldsymbol{q} \neq \mathbf{0}} \beta_{\boldsymbol{q}}^{\dagger} \beta_{\boldsymbol{q}}\right)^{2} \approx N_{\beta}^{2}-2 N_{\beta} \sum_{\boldsymbol{q} \neq \mathbf{0}} \beta_{\boldsymbol{q}}^{\dagger} \beta_{\boldsymbol{q}}=N_{\mathrm{m}}^{2}-2 N_{\mathrm{m}} \sum_{\boldsymbol{q} \neq \mathbf{0}} \beta_{\boldsymbol{q}}^{\dagger} \beta_{\boldsymbol{q}}, \\
& n_{\mathbf{0} \beta} \sum_{\boldsymbol{q} \neq \mathbf{0}} \beta_{\boldsymbol{q}}^{\dagger} \beta_{\boldsymbol{q}} \approx N_{\beta} \sum_{\boldsymbol{q} \neq \mathbf{0}} \beta_{\boldsymbol{q}}^{\dagger} \beta_{\boldsymbol{q}}=N_{\mathrm{m}} \sum_{\boldsymbol{q} \neq \mathbf{0}} \beta_{\boldsymbol{q}}^{\dagger} \beta_{\boldsymbol{q}},
\end{aligned}
$$

for the derivation of Eq. C3,

$$
\begin{aligned}
& n_{\mathbf{0} \alpha} n_{\mathbf{0} \beta} \approx N_{\alpha} N_{\beta}-N_{\alpha} \sum_{\boldsymbol{q} \neq \mathbf{0}} \beta_{\boldsymbol{q}}^{\dagger} \beta_{\boldsymbol{q}}-N_{\beta} \sum_{\boldsymbol{q} \neq \mathbf{0}} \alpha_{\boldsymbol{q}}^{\dagger} \alpha_{\boldsymbol{q}}=N_{\mathrm{m}}^{2}-N_{\mathrm{m}} \sum_{\boldsymbol{q} \neq \mathbf{0}}\left(\alpha_{\boldsymbol{q}}^{\dagger} \alpha_{\boldsymbol{q}}+\beta_{\boldsymbol{q}}^{\dagger} \beta_{\boldsymbol{q}}\right), \\
& n_{\mathbf{0} \beta} \sum_{\boldsymbol{q} \neq \mathbf{0}} \alpha_{\boldsymbol{q}}^{\dagger} \alpha_{\boldsymbol{q}} \approx N_{\beta} \sum_{\boldsymbol{q} \neq \mathbf{0}} \alpha_{\boldsymbol{q}}^{\dagger} \alpha_{\boldsymbol{q}}=N_{\mathrm{m}} \sum_{\boldsymbol{q} \neq \mathbf{0}} \alpha_{\boldsymbol{q}}^{\dagger} \alpha_{\boldsymbol{q}}, \\
& n_{\mathbf{0} \alpha} \sum_{\boldsymbol{q} \neq \mathbf{0}} \beta_{\boldsymbol{q}}^{\dagger} \beta_{\boldsymbol{q}} \approx N_{\alpha} \sum_{\boldsymbol{q} \neq \mathbf{0}} \beta_{\boldsymbol{q}}^{\dagger} \beta_{\boldsymbol{q}}=N_{\mathrm{m}} \sum_{\boldsymbol{q} \neq \mathbf{0}} \beta_{\boldsymbol{q}}^{\dagger} \beta_{\boldsymbol{q}}, \\
& \sqrt{n_{\mathbf{0} \alpha}} \sqrt{n_{\mathbf{0} \beta}} \sum_{\boldsymbol{q} \neq \mathbf{0}}\left(\alpha_{\boldsymbol{q}} \beta_{\boldsymbol{q}}+\alpha_{\boldsymbol{q}}^{\dagger} \beta_{\boldsymbol{q}}^{\dagger}\right) \approx \sqrt{N_{\alpha} N_{\beta}} \sum_{\boldsymbol{q} \neq \mathbf{0}}\left(\alpha_{\boldsymbol{q}} \beta_{\boldsymbol{q}}+\alpha_{\boldsymbol{q}}^{\dagger} \beta_{\boldsymbol{q}}^{\dagger}\right)=N_{\mathrm{m}} \sum_{\boldsymbol{q} \neq \mathbf{0}}\left(\alpha_{\boldsymbol{q}} \beta_{\boldsymbol{q}}+\alpha_{\boldsymbol{q}}^{\dagger} \beta_{\boldsymbol{q}}^{\dagger}\right),
\end{aligned}
$$

for the derivation of Eq. C4,

$$
\begin{aligned}
n_{\mathbf{0} \alpha} \sqrt{n_{\mathbf{0} \alpha}} \sqrt{n_{\mathbf{0} \beta}} & \approx\left(N_{\alpha}-\sum_{\boldsymbol{q} \neq \mathbf{0}} \alpha_{\boldsymbol{q}}^{\dagger} \alpha_{\boldsymbol{q}}\right)\left[\sqrt{N_{\alpha} N_{\beta}}-\frac{1}{2} \sum_{\boldsymbol{q} \neq \mathbf{0}}\left(\alpha_{\boldsymbol{q}}^{\dagger} \alpha_{\boldsymbol{q}}+\beta_{\boldsymbol{q}}^{\dagger} \beta_{\boldsymbol{q}}\right)\right] \\
& \approx N_{\alpha} \sqrt{N_{\alpha} N_{\beta}}-\sqrt{N_{\alpha} N_{\beta}} \sum_{\boldsymbol{q} \neq \mathbf{0}} \alpha_{\boldsymbol{q}}^{\dagger} \alpha_{\boldsymbol{q}}-\frac{1}{2} N_{\alpha} \sum_{\boldsymbol{q} \neq \mathbf{0}}\left(\alpha_{\boldsymbol{q}}^{\dagger} \alpha_{\boldsymbol{q}}+\beta_{\boldsymbol{q}}^{\dagger} \beta_{\boldsymbol{q}}\right) \\
& =N_{\mathrm{m}}^{2}-\frac{1}{2} N_{\mathrm{m}} \sum_{\boldsymbol{q} \neq \mathbf{0}}\left(3 \alpha_{\boldsymbol{q}}^{\dagger} \alpha_{\boldsymbol{q}}+\beta_{\boldsymbol{q}}^{\dagger} \beta_{\boldsymbol{q}}\right), \\
\sqrt{n_{\mathbf{0} \alpha}} \sqrt{n_{\mathbf{0} \beta}} \sum_{\boldsymbol{q} \neq \mathbf{0}} \alpha_{\boldsymbol{q}}^{\dagger} \alpha_{\boldsymbol{q}} & \approx \sqrt{N_{\alpha} N_{\beta}} \sum_{\boldsymbol{q} \neq \mathbf{0}} \alpha_{\boldsymbol{q}}^{\dagger} \alpha_{\boldsymbol{q}}=N_{\mathrm{m}} \sum_{\boldsymbol{q} \neq \mathbf{0}} \alpha_{\boldsymbol{q}}^{\dagger} \alpha_{\boldsymbol{q}}, \\
n_{\mathbf{0} \alpha} \sum_{\boldsymbol{q} \neq \mathbf{0}}\left(\alpha_{\boldsymbol{q}} \beta_{\boldsymbol{q}}+\alpha_{\boldsymbol{q}}^{\dagger} \beta_{\boldsymbol{q}}^{\dagger}\right) & \approx N_{\alpha} \sum_{\boldsymbol{q} \neq \mathbf{0}}\left(\alpha_{\boldsymbol{q}} \beta_{\boldsymbol{q}}+\alpha_{\boldsymbol{q}}^{\dagger} \beta_{\boldsymbol{q}}^{\dagger}\right)=N_{\mathrm{m}} \sum_{\boldsymbol{q} \neq \mathbf{0}}\left(\alpha_{\boldsymbol{q}} \beta_{\boldsymbol{q}}+\alpha_{\boldsymbol{q}}^{\dagger} \beta_{\boldsymbol{q}}^{\dagger}\right),
\end{aligned}
$$

for the derivation of Eq. C5, and

$$
\begin{aligned}
n_{\mathbf{0} \beta} \sqrt{n_{\mathbf{0} \alpha}} \sqrt{n_{\mathbf{0} \beta}} & \approx\left(N_{\beta}-\sum_{\boldsymbol{q} \neq \mathbf{0}} \beta_{\boldsymbol{q}}^{\dagger} \beta_{\boldsymbol{q}}\right)\left[\sqrt{N_{\alpha} N_{\beta}}-\frac{1}{2} \sum_{\boldsymbol{q} \neq \mathbf{0}}\left(\alpha_{\boldsymbol{q}}^{\dagger} \alpha_{\boldsymbol{q}}+\beta_{\boldsymbol{q}}^{\dagger} \beta_{\boldsymbol{q}}\right)\right] \\
& \approx N_{\beta} \sqrt{N_{\alpha} N_{\beta}}-\sqrt{N_{\alpha} N_{\beta}} \sum_{\boldsymbol{q} \neq \mathbf{0}} \beta_{\boldsymbol{q}}^{\dagger} \beta_{\boldsymbol{q}}-\frac{1}{2} N_{\beta} \sum_{\boldsymbol{q} \neq \mathbf{0}}\left(\alpha_{\boldsymbol{q}}^{\dagger} \alpha_{\boldsymbol{q}}+\beta_{\boldsymbol{q}}^{\dagger} \beta_{\boldsymbol{q}}\right) \\
& =N_{\mathrm{m}}^{2}-\frac{1}{2} N_{\mathrm{m}} \sum_{\boldsymbol{q} \neq \mathbf{0}}\left(\alpha_{\boldsymbol{q}}^{\dagger} \alpha_{\boldsymbol{q}}+3 \beta_{\boldsymbol{q}}^{\dagger} \beta_{\boldsymbol{q}}\right), \\
\sqrt{n_{\mathbf{0} \alpha}} \sqrt{n_{\mathbf{0} \beta}} \sum_{\boldsymbol{q} \neq \mathbf{0}} \beta_{\boldsymbol{q}}^{\dagger} \beta_{\boldsymbol{q}} & \approx \sqrt{N_{\alpha} N_{\beta}} \sum_{\boldsymbol{q} \neq \mathbf{0}} \beta_{\boldsymbol{q}}^{\dagger} \beta_{\boldsymbol{q}}=N_{\mathrm{m}} \sum_{\boldsymbol{q} \neq \mathbf{0}} \beta_{\boldsymbol{q}}^{\dagger} \beta_{\boldsymbol{q}}, \\
n_{\mathbf{0} \beta} \sum_{\boldsymbol{q} \neq \mathbf{0}}\left(\alpha_{\boldsymbol{q}} \beta_{\boldsymbol{q}}+\alpha_{\boldsymbol{q}}^{\dagger} \beta_{\boldsymbol{q}}^{\dagger}\right) & \approx N_{\beta} \sum_{\boldsymbol{q} \neq \mathbf{0}}\left(\alpha_{\boldsymbol{q}} \beta_{\boldsymbol{q}}+\alpha_{\boldsymbol{q}}^{\dagger} \beta_{\boldsymbol{q}}^{\dagger}\right)=N_{\mathrm{m}} \sum_{\boldsymbol{q} \neq \mathbf{0}}\left(\alpha_{\boldsymbol{q}} \beta_{\boldsymbol{q}}+\alpha_{\boldsymbol{q}}^{\dagger} \beta_{\boldsymbol{q}}^{\dagger}\right),
\end{aligned}
$$


for the derivation of Eq. (C6). (As described above, $N_{\alpha}=N_{\beta} \equiv N_{\mathrm{m}}$ for $h=0$.) Since the operator terms in Eqs. (C2) - C6 are relevant to the interaction effects on the stability of the magnon BEC, we consider only the operator parts in the following. Therefore we have

$$
\begin{aligned}
& H_{\alpha \alpha \alpha \alpha}^{(0)}+H_{\beta \beta \beta \beta}^{(0)}=\frac{N_{\mathrm{m}}}{N} \sum_{\boldsymbol{q} \neq \mathbf{0}} \Gamma_{\text {intra }}^{(0)}(\boldsymbol{q})\left(\alpha_{\boldsymbol{q}}^{\dagger} \alpha_{\boldsymbol{q}}+\beta_{\boldsymbol{q}}^{\dagger} \beta_{\boldsymbol{q}}\right), \\
& H_{\alpha \alpha \beta \beta}^{(0)}=\frac{N_{\mathrm{m}}}{N} \sum_{\boldsymbol{q} \neq \mathbf{0}} \Gamma_{\text {inter } 1}^{(0)}(\boldsymbol{q})\left(\alpha_{\boldsymbol{q}}^{\dagger} \alpha_{\boldsymbol{q}}+\beta_{\boldsymbol{q}}^{\dagger} \beta_{\boldsymbol{q}}\right)+\frac{N_{\mathrm{m}}}{N} \sum_{\boldsymbol{q} \neq \mathbf{0}} \Gamma_{\text {inter } 2}^{(0)}(\boldsymbol{q})\left(\alpha_{\boldsymbol{q}} \beta_{\boldsymbol{q}}+\alpha_{\boldsymbol{q}}^{\dagger} \beta_{\boldsymbol{q}}^{\dagger}\right), \\
& H_{\alpha \alpha \alpha \beta}^{(0)}+H_{\alpha \beta \beta \beta}^{(0)}=\frac{N_{\mathrm{m}}}{N} \sum_{\boldsymbol{q} \neq \mathbf{0}} \Gamma_{\text {inter } 3}^{(0)}(\boldsymbol{q})\left(\alpha_{\boldsymbol{q}}^{\dagger} \alpha_{\boldsymbol{q}}+\beta_{\boldsymbol{q}}^{\dagger} \beta_{\boldsymbol{q}}\right)+\frac{N_{\mathrm{m}}}{N} \sum_{\boldsymbol{q} \neq \mathbf{0}} \Gamma_{\text {inter2 }}^{(0)}(\boldsymbol{q})\left(\alpha_{\boldsymbol{q}} \beta_{\boldsymbol{q}}+\alpha_{\boldsymbol{q}}^{\dagger} \beta_{\boldsymbol{q}}^{\dagger}\right) .
\end{aligned}
$$

Here we have introduced the following quantities:

$$
\begin{aligned}
\Gamma_{\text {intra }}^{(0)}(\boldsymbol{q})= & -8 J(\mathbf{0}) c_{\mathbf{0}} s_{\mathbf{0}}\left(c_{\mathbf{0}}^{2}+s_{\mathbf{0}}^{2}-2 c_{\mathbf{0}} s_{\mathbf{0}}\right)-4 J(\mathbf{0})\left(c_{\boldsymbol{q}}^{2} s_{\mathbf{0}}^{2}+c_{\mathbf{0}}^{2} s_{\boldsymbol{q}}^{2}\right)-8 J(\boldsymbol{q}) c_{\boldsymbol{q}} s_{\boldsymbol{q}} c_{\mathbf{0}} s_{\mathbf{0}}+4 J(\mathbf{0}) c_{\mathbf{0}} s_{\mathbf{0}}\left(c_{\boldsymbol{q}}^{2}+s_{\boldsymbol{q}}^{2}\right) \\
& +4 J(\boldsymbol{q}) c_{\boldsymbol{q}} s_{\boldsymbol{q}}\left(c_{\mathbf{0}}^{2}+s_{\mathbf{0}}^{2}\right), \\
\Gamma_{\text {inter } 1}^{(0)}(\boldsymbol{q})= & 8 J(\mathbf{0})\left(c_{\mathbf{0}}^{2}+s_{\mathbf{0}}^{2}-c_{\mathbf{0}} s_{\mathbf{0}}\right)\left(c_{\mathbf{0}}^{2}+s_{\mathbf{0}}^{2}-2 c_{\mathbf{0}} s_{\mathbf{0}}\right)-4 J(\mathbf{0})\left[\left(c_{\boldsymbol{q}}^{2} c_{\mathbf{0}}^{2}+s_{\boldsymbol{q}}^{2} s_{\mathbf{0}}^{2}\right)-c_{\mathbf{0}} s_{\mathbf{0}}\left(c_{\boldsymbol{q}}^{2}+s_{\boldsymbol{q}}^{2}\right)\right] \\
& +4 J(\boldsymbol{q}) c_{\boldsymbol{q}} s_{\boldsymbol{q}}\left(c_{\mathbf{0}}^{2}+s_{\mathbf{0}}^{2}-2 c_{\mathbf{0}} s_{\mathbf{0}}\right), \\
\Gamma_{\text {inter2}}^{(0)}(\boldsymbol{q})= & -4\left(c_{\mathbf{0}}^{2}+s_{\mathbf{0}}^{2}-2 c_{\mathbf{0}} s_{\mathbf{0}}\right)\left[J(\boldsymbol{q})\left(c_{\boldsymbol{q}}^{2}+s_{\boldsymbol{q}}^{2}\right)-2 J(\mathbf{0}) c_{\boldsymbol{q}} s_{\boldsymbol{q}}\right], \\
\Gamma_{\text {inter3 }}^{(0)}(\boldsymbol{q})= & 8 J(\mathbf{0})-4\left(c_{\mathbf{0}}^{2}+s_{\mathbf{0}}^{2}-2 c_{\mathbf{0}} s_{\mathbf{0}}\right)\left[8 J(\mathbf{0}) c_{\mathbf{0}} s_{\mathbf{0}}+J(\mathbf{0})\left(c_{\boldsymbol{q}}^{2}+s_{\boldsymbol{q}}^{2}\right)-2 J(\boldsymbol{q}) c_{\boldsymbol{q}} s_{\boldsymbol{q}}\right] .
\end{aligned}
$$

Since the low- $\boldsymbol{q}$ magnons of the $\alpha$ and $\beta$ bands describe the low-energy noncondensates in the magnon BEC of the antiferromagnet for $h=0$, we estimate $\Gamma_{\text {intra }}^{(0)}(\boldsymbol{q}), \Gamma_{\text {inter } 1}^{(0)}(\boldsymbol{q}), \Gamma_{\text {inter2 }}^{(0)}(\boldsymbol{q})$, and $\Gamma_{\text {inter3 }}^{(0)}(\boldsymbol{q})$ in the limit $|\boldsymbol{q}| \rightarrow 0$. To estimate them, we use the following identities:

$$
\begin{aligned}
& c_{\boldsymbol{q}}^{2}-s_{\boldsymbol{q}}^{2}=1, \\
& c_{\boldsymbol{q}}^{2}+s_{\boldsymbol{q}}^{2}=\cosh 2 \theta_{\boldsymbol{q}}, \\
& 2 c_{\boldsymbol{q}} s_{\boldsymbol{q}}=\sinh 2 \theta_{\boldsymbol{q}} .
\end{aligned}
$$

In addition, $\cosh 2 \theta_{\boldsymbol{q}}$ and $\sinh 2 \theta_{\boldsymbol{q}}$ are given for our antiferromagnet by

$$
\begin{aligned}
& \cosh 2 \theta_{\boldsymbol{q}}=\frac{J(\mathbf{0})+K}{\sqrt{(J(\mathbf{0})+K)^{2}-J(\boldsymbol{q})^{2}}} \sim \frac{J(\mathbf{0})+K}{\sqrt{2 J(\mathbf{0}) K}}, \\
& \sinh 2 \theta_{\boldsymbol{q}}=\frac{J(\boldsymbol{q})}{\sqrt{(J(\mathbf{0})+K)^{2}-J(\boldsymbol{q})^{2}}} \sim \frac{J(\mathbf{0})}{\sqrt{2 J(\mathbf{0}) K}} .
\end{aligned}
$$

(In the above limiting expressions we have considered only the leading terms in the limit $|\boldsymbol{q}| \rightarrow 0$ for $J \gg K$.) By 
using Eqs. $\mathrm{C} 28-\mathrm{C} 32$ and retaining the leading terms in the limit $|\boldsymbol{q}| \rightarrow 0$ for $J \gg K$, we obtain

$$
\begin{aligned}
& -8 J(\mathbf{0}) c_{\mathbf{0}} s_{\mathbf{0}}\left(c_{\mathbf{0}}^{2}+s_{\mathbf{0}}^{2}-2 c_{\mathbf{0}} s_{\mathbf{0}}\right) \sim-4 J(\mathbf{0}) \sqrt{\frac{K}{2 J(\mathbf{0})}} \sqrt{\frac{J(\mathbf{0})}{2 K}}=-2 J(\mathbf{0}), \\
& -4 J(\mathbf{0})\left(c_{\boldsymbol{q}}^{2} s_{\mathbf{0}}^{2}+c_{\mathbf{0}}^{2} s_{\boldsymbol{q}}^{2}\right)+4 J(\mathbf{0}) c_{\mathbf{0}} s_{\mathbf{0}}\left(c_{\boldsymbol{q}}^{2}+s_{\boldsymbol{q}}^{2}\right) \sim 2 J(\mathbf{0})-2 \sqrt{\frac{J(\mathbf{0})}{2 K}} \sqrt{\frac{K}{2 J(\mathbf{0})}}=J(\mathbf{0}), \\
& -8 J(\boldsymbol{q}) c_{\boldsymbol{q}} s_{\boldsymbol{q}} c_{\mathbf{0}} s_{\mathbf{0}}+4 J(\boldsymbol{q}) c_{\boldsymbol{q}} s_{\boldsymbol{q}}\left(c_{\mathbf{0}}^{2}+s_{\mathbf{0}}^{2}\right) \sim 2 J(\mathbf{0}) \sqrt{\frac{J(\mathbf{0})}{2 K}} \sqrt{\frac{K}{2 J(\mathbf{0})}}=J(\mathbf{0}), \\
& 8 J(\mathbf{0})\left(c_{\mathbf{0}}^{2}+s_{\mathbf{0}}^{2}-c_{\mathbf{0}} s_{\mathbf{0}}\right)\left(c_{\mathbf{0}}^{2}+s_{\mathbf{0}}^{2}-2 c_{\mathbf{0}} s_{\mathbf{0}}\right) \sim 8 J(\mathbf{0}) \frac{1}{2} J(\mathbf{0}) \frac{1}{\sqrt{2 J(\mathbf{0}) K}} \sqrt{\frac{K}{2 J(\mathbf{0})}}=2 J(\mathbf{0}), \\
& -4 J(\mathbf{0})\left[\left(c_{\boldsymbol{q}}^{2} c_{\mathbf{0}}^{2}+s_{\boldsymbol{q}}^{2} s_{\mathbf{0}}^{2}\right)-c_{\mathbf{0}} s_{\mathbf{0}}\left(c_{\boldsymbol{q}}^{2}+s_{\boldsymbol{q}}^{2}\right)\right] \sim-2 J(\mathbf{0})-2 J(\mathbf{0}) \sqrt{\frac{J(\mathbf{0})}{2 K}} \sqrt{\frac{K}{2 J(\mathbf{0})}}=-3 J(\mathbf{0}), \\
& 4 J(\boldsymbol{q}) c_{\boldsymbol{q}} s_{\boldsymbol{q}}\left(c_{\mathbf{0}}^{2}+s_{\mathbf{0}}^{2}-2 c_{\mathbf{0}} s_{\mathbf{0}}\right) \sim 2 J(\mathbf{0}) \frac{J(\mathbf{0})}{\sqrt{2 J(\mathbf{0}) K}} \sqrt{\frac{K}{2 J(\mathbf{0})}}=J(\mathbf{0}), \\
& -4\left(c_{\mathbf{0}}^{2}+s_{\mathbf{0}}^{2}-2 c_{\mathbf{0}} s_{\mathbf{0}}\right)\left[J(\boldsymbol{q})\left(c_{\boldsymbol{q}}^{2}+s_{\boldsymbol{q}}^{2}\right)-2 J(\mathbf{0}) c_{\boldsymbol{q}} s_{\boldsymbol{q}}\right] \sim-4 \sqrt{\frac{K}{2 J(\mathbf{0})}} \frac{J(\mathbf{0}) K}{\sqrt{2 J(\mathbf{0}) K}} \approx 0, \\
& -4\left(c_{\mathbf{0}}^{2}+s_{\mathbf{0}}^{2}-2 c_{\mathbf{0}} s_{\mathbf{0}}\right)\left[8 J(\mathbf{0}) c_{\mathbf{0}} s_{\mathbf{0}}+J(\mathbf{0})\left(c_{\boldsymbol{q}}^{2}+s_{\boldsymbol{q}}^{2}\right)-2 J(\boldsymbol{q}) c_{\boldsymbol{q}} s_{\boldsymbol{q}}\right] \sim-16 J(\mathbf{0}) \sqrt{\frac{K}{2 J(\mathbf{0})}} \sqrt{\frac{J(\mathbf{0})}{2 K}}=-8 J(\mathbf{0}) .
\end{aligned}
$$

(We have considered only the terms of order $J$ and neglected the higher-order terms, such as the term of order $K$, because we have considered the case for $J \gg K$.) Combining Eqs. (C33)-(C40) with Eqs. (C24)-(C27), we have

$$
\begin{aligned}
& \Gamma_{\text {intra }}^{(0)}(\boldsymbol{q}) \sim-2 J(\mathbf{0})+J(\mathbf{0})+J(\mathbf{0})=0, \\
& \Gamma_{\text {inter1 }}^{(0)}(\boldsymbol{q}) \sim 2 J(\mathbf{0})-3 J(\mathbf{0})+J(\mathbf{0})=0, \\
& \Gamma_{\text {inter2 }}^{(0)}(\boldsymbol{q}) \sim 0, \\
& \Gamma_{\text {inter3 }}^{(0)}(\boldsymbol{q}) \sim 8 J(\mathbf{0})-8 J(\mathbf{0})=0 .
\end{aligned}
$$

Therefore we find that the coefficients of the mean-field interaction terms which arise from the terms of $H_{\text {int }}$ for $\boldsymbol{q}=\boldsymbol{q}^{\prime}=\mathbf{0}$, for $\boldsymbol{q}=\mathbf{0}, \boldsymbol{q}^{\prime} \neq \mathbf{0}$, and for $\boldsymbol{q} \neq \mathbf{0}, \boldsymbol{q}^{\prime}=\mathbf{0}$ become zero within the leading order. Note that this property holds even for $h \neq 0$ because $\cosh 2 \theta_{\boldsymbol{q}}$ and $\sinh 2 \theta_{\boldsymbol{q}}$ are independent of $h$ [see Eqs. (C31) and (C32)].

\section{Appendix D: Derivation of Eqs. (8) and (9)}

We derive Eqs. (8) and (9), i.e., the mean-field interaction terms which arise from the terms of $H_{\text {int }}=H_{\text {intra }}+H_{\text {inter }}$ for $\boldsymbol{q} \neq \mathbf{0}, \boldsymbol{q}^{\prime} \neq \mathbf{0}$. In this derivation we replace two of the four operators for each term in Eqs. (B1) - B5 by the corresponding expectation value; for $\boldsymbol{q} \neq \mathbf{0}, \boldsymbol{q}^{\prime} \neq \mathbf{0}$, the expectation values of two operators satisfy $\left\langle\alpha_{\boldsymbol{q}}^{\dagger} \alpha_{\boldsymbol{q}^{\prime}}\right\rangle=\delta_{\boldsymbol{q}, \boldsymbol{q}^{\prime}} n_{\boldsymbol{q} \alpha}$, $\left\langle\beta_{\boldsymbol{q}}^{\dagger} \beta_{\boldsymbol{q}^{\prime}}\right\rangle=\delta_{\boldsymbol{q}, \boldsymbol{q}^{\prime}} n_{\boldsymbol{q} \beta},\left\langle\alpha_{\boldsymbol{q}} \beta_{\boldsymbol{q}^{\prime}}\right\rangle=0$, and $\left\langle\alpha_{\boldsymbol{q}}^{\dagger} \beta_{\boldsymbol{q}^{\prime}}^{\dagger}\right\rangle=0$. Thus, for example, the leading terms which arise from the first term of $V_{1}$ in Eq. (B1) for $\boldsymbol{q} \neq \mathbf{0}, \boldsymbol{q}^{\prime} \neq \mathbf{0}$ become

$$
-\frac{2}{N} \sum_{\boldsymbol{q}, \boldsymbol{q}^{\prime} \neq \mathbf{0}} J(\mathbf{0}) c_{\boldsymbol{q}^{2}}^{2} s_{\boldsymbol{q}^{\prime}}^{2}\left(n_{\boldsymbol{q} \alpha} \alpha_{\boldsymbol{q}^{\prime}}^{\dagger} \alpha_{\boldsymbol{q}^{\prime}}+n_{\boldsymbol{q}^{\prime} \alpha} \alpha_{\boldsymbol{q}}^{\dagger} \alpha_{\boldsymbol{q}}\right)
$$

Here we have considered only the terms proportional to $n_{\boldsymbol{q} \alpha} / N$ or $n_{\boldsymbol{q}^{\prime} \alpha} / N$ and neglected the terms proportional to $1 / N$ because $n_{\boldsymbol{q} \alpha} \gg 1$ for small $\boldsymbol{q}$. We can similarly derive the leading terms which arise from the other terms. After some calculation we can write the mean-field interaction terms which arise from the terms of $H_{\alpha \alpha \alpha \alpha}, H_{\beta \beta \beta \beta}, H_{\alpha \alpha \beta \beta}$, 
$H_{\alpha \alpha \alpha \beta}$, and $H_{\alpha \beta \beta \beta}$ for $\boldsymbol{q} \neq \mathbf{0}, \boldsymbol{q}^{\prime} \neq \mathbf{0}$ as follows:

$$
\begin{aligned}
& H_{\alpha \alpha \alpha \alpha}=\frac{1}{N} \sum_{\boldsymbol{q}, \boldsymbol{q}^{\prime} \neq \mathbf{0}} \Gamma_{\mathrm{intra1}}\left(\boldsymbol{q}, \boldsymbol{q}^{\prime}\right) n_{\boldsymbol{q}^{\prime} \alpha} \alpha_{\boldsymbol{q}}^{\dagger} \alpha_{\boldsymbol{q}}, \\
& H_{\beta \beta \beta \beta}=\frac{1}{N} \sum_{\boldsymbol{q}, \boldsymbol{q}^{\prime} \neq \mathbf{0}} \Gamma_{\mathrm{intra} 1}\left(\boldsymbol{q}, \boldsymbol{q}^{\prime}\right) n_{\boldsymbol{q}^{\prime} \beta} \beta_{\boldsymbol{q}}^{\dagger} \beta_{\boldsymbol{q}}, \\
& H_{\alpha \alpha \beta \beta}=\frac{1}{N} \sum_{\boldsymbol{q}, \boldsymbol{q}^{\prime} \neq \mathbf{0}} \Gamma_{\mathrm{inter} 1}\left(\boldsymbol{q}, \boldsymbol{q}^{\prime}\right)\left(n_{\boldsymbol{q}^{\prime} \beta} \alpha_{\boldsymbol{q}}^{\dagger} \alpha_{\boldsymbol{q}}+n_{\boldsymbol{q}^{\prime} \alpha} \beta_{\boldsymbol{q}}^{\dagger} \beta_{\boldsymbol{q}}\right)+\frac{1}{N} \sum_{\boldsymbol{q} \neq \mathbf{0}} \Gamma_{\mathrm{inter} 2}(\boldsymbol{q})\left(n_{\boldsymbol{q} \beta} \alpha_{\boldsymbol{q}}^{\dagger} \alpha_{\boldsymbol{q}}+n_{\boldsymbol{q} \alpha} \beta_{\boldsymbol{q}}^{\dagger} \beta_{\boldsymbol{q}}\right), \\
& H_{\alpha \alpha \alpha \beta}=\frac{1}{N} \sum_{\boldsymbol{q}, \boldsymbol{q}^{\prime} \neq \mathbf{0}} \Gamma_{\mathrm{inter} 3}\left(\boldsymbol{q}, \boldsymbol{q}^{\prime}\right) n_{\boldsymbol{q}^{\prime} \alpha}\left(\alpha_{\boldsymbol{q}} \beta_{\boldsymbol{q}}+\alpha_{\boldsymbol{q}}^{\dagger} \beta_{\boldsymbol{q}}^{\dagger}\right)+\frac{1}{N} \sum_{\boldsymbol{q} \neq \mathbf{0}} \Gamma_{\mathrm{inter} 4}(\boldsymbol{q}) n_{\boldsymbol{q} \alpha}\left(\alpha_{\boldsymbol{q}} \beta_{\boldsymbol{q}}+\alpha_{\boldsymbol{q}}^{\dagger} \beta_{\boldsymbol{q}}^{\dagger}\right), \\
& H_{\alpha \beta \beta \beta}=\frac{1}{N} \sum_{\boldsymbol{q}, \boldsymbol{q}^{\prime} \neq \mathbf{0}} \Gamma_{\mathrm{inter} 3}\left(\boldsymbol{q}, \boldsymbol{q}^{\prime}\right) n_{\boldsymbol{q}^{\prime} \beta}\left(\alpha_{\boldsymbol{q}} \beta_{\boldsymbol{q}}+\alpha_{\boldsymbol{q}}^{\dagger} \beta_{\boldsymbol{q}}^{\dagger}\right)+\frac{1}{N} \sum_{\boldsymbol{q} \neq \mathbf{0}} \Gamma_{\mathrm{inter} 4}(\boldsymbol{q}) n_{\boldsymbol{q} \beta}\left(\alpha_{\boldsymbol{q}} \beta_{\boldsymbol{q}}+\alpha_{\boldsymbol{q}}^{\dagger} \beta_{\boldsymbol{q}}^{\dagger}\right),
\end{aligned}
$$

where we have used Eqs. (10)- (14). Combining Eqs. (D2)-(D6) with $H_{\text {intra }}=H_{\alpha \alpha \alpha \alpha}+H_{\beta \beta \beta \beta}$ and $H_{\text {inter }}=$ $H_{\alpha \alpha \beta \beta}+H_{\alpha \alpha \alpha \beta}+H_{\alpha \beta \beta \beta}$, we obtain Eqs. (8) and (9).

\section{Appendix E: Derivation of Eqs. (15)- 19}

We derive Eqs. $15-(19)$ by estimating the leading-order terms of Eqs. (10)- 14 in the limit $|\boldsymbol{q}|,\left|\boldsymbol{q}^{\prime}\right| \rightarrow 0$. We can estimate Eqs. (10)-(14), i.e., the coefficients of Eqs. (8) and (9), in the limit $|\boldsymbol{q}|,\left|\boldsymbol{q}^{\prime}\right| \rightarrow 0$ in the same way as the estimation of $\Gamma_{\text {intra }}^{(0)}(\boldsymbol{q}), \Gamma_{\text {inter1 }}^{(0)}(\boldsymbol{q}), \Gamma_{\text {inter2 }}^{(0)}(\boldsymbol{q})$, and $\Gamma_{\text {inter3 }}^{(0)}(\boldsymbol{q})$ [for the latter estimation see Appendix C, in particular, the derivation of Eqs. C33 - C40 ]. As a result, we obtain

$$
\begin{aligned}
& \Gamma_{\text {intra1 }}\left(\boldsymbol{q}, \boldsymbol{q}^{\prime}\right)=2 J(\mathbf{0})-2 J(\mathbf{0}) \cosh 2 \theta_{\boldsymbol{q}} \cosh 2 \theta_{\boldsymbol{q}^{\prime}}-2 J\left(\boldsymbol{q}-\boldsymbol{q}^{\prime}\right) \sinh 2 \theta_{\boldsymbol{q}} \sinh 2 \theta_{\boldsymbol{q}^{\prime}}+2 J(\boldsymbol{q}) \sinh 2 \theta_{\boldsymbol{q}} \cosh 2 \theta_{\boldsymbol{q}^{\prime}} \\
& +2 J\left(\boldsymbol{q}^{\prime}\right) \cosh 2 \theta_{\boldsymbol{q}} \sinh 2 \theta_{\boldsymbol{q}^{\prime}} \\
& \sim 2 J(\mathbf{0})\left[1-\frac{J(\mathbf{0})^{2}+2 J(\mathbf{0}) K}{2 J(\mathbf{0}) K}-\frac{J(\mathbf{0})^{2}}{2 J(\mathbf{0}) K}+\frac{J(\mathbf{0})^{2}+J(\mathbf{0}) K}{2 J(\mathbf{0}) K}+\frac{J(\mathbf{0})^{2}+J(\mathbf{0}) K}{2 J(\mathbf{0}) K}\right] \\
& =2 J(\mathbf{0}) \text {, } \\
& \Gamma_{\text {inter } 1}\left(\boldsymbol{q}, \boldsymbol{q}^{\prime}\right)=-4 J(\mathbf{0})+\Gamma_{\text {intra1 }}\left(\boldsymbol{q}, \boldsymbol{q}^{\prime}\right) \\
& \sim-2 J(\mathbf{0}), \\
& \Gamma_{\text {inter2 }}(\boldsymbol{q})=-4 \cosh 2 \theta_{\boldsymbol{q}}\left[J(\mathbf{0}) \cosh 2 \theta_{\boldsymbol{q}}-J(\boldsymbol{q}) \sinh 2 \theta_{\boldsymbol{q}}\right] \\
& \sim-4 J(\mathbf{0})+2 J(\mathbf{0}) \\
& =-2 J(\mathbf{0}) \text {, } \\
& \Gamma_{\text {inter } 3}\left(\boldsymbol{q}, \boldsymbol{q}^{\prime}\right)=2 J(\mathbf{0}) \sinh 2 \theta_{\boldsymbol{q}} \cosh 2 \theta_{\boldsymbol{q}^{\prime}}+2 J\left(\boldsymbol{q}-\boldsymbol{q}^{\prime}\right) \sinh 2 \theta_{\boldsymbol{q}^{\prime}} \cosh 2 \theta_{\boldsymbol{q}}-2 J(\boldsymbol{q}) \cosh 2 \theta_{\boldsymbol{q}} \cosh 2 \theta_{\boldsymbol{q}^{\prime}} \\
& -2 J\left(\boldsymbol{q}^{\prime}\right) \sinh 2 \theta_{\boldsymbol{q}^{\prime}} \sinh 2 \theta_{\boldsymbol{q}} \\
& \sim\left[J(\mathbf{0}) \frac{J(\mathbf{0})}{K}+J(\mathbf{0})\right]+\left[J(\mathbf{0}) \frac{J(\mathbf{0})}{K}+J(\mathbf{0})\right]-\left[J(\mathbf{0}) \frac{J(\mathbf{0})}{K}+2 J(\mathbf{0})\right]-J(\mathbf{0}) \frac{J(\mathbf{0})}{K} \\
& =0 \text {, } \\
& \Gamma_{\text {inter } 4}(\boldsymbol{q})=4 J(\mathbf{0}) \sinh 2 \theta_{\boldsymbol{q}} \cosh 2 \theta_{\boldsymbol{q}}-2 J(\boldsymbol{q})\left(\cosh 2 \theta_{\boldsymbol{q}}\right)^{2}-2 J(\boldsymbol{q})\left(\sinh 2 \theta_{\boldsymbol{q}}\right)^{2} \\
& \sim\left[2 J(\mathbf{0}) \frac{J(\mathbf{0})}{K}+2 J(\mathbf{0})\right]-\left[J(\mathbf{0}) \frac{J(\mathbf{0})}{K}+2 J(\mathbf{0})\right]-J(\mathbf{0}) \frac{J(\mathbf{0})}{K} \\
& =0 \text {. }
\end{aligned}
$$

Note that Eqs. (D5) and (D6) with Eqs. (E4) and (E5) show that the contributions from $H_{\alpha \alpha \alpha \beta}$ and $H_{\alpha \beta \beta \beta}$, corresponding to the mean-field interaction terms including $\alpha_{\boldsymbol{q}} \beta_{\boldsymbol{q}}$ and $\alpha_{\boldsymbol{q}}^{\dagger} \beta_{\boldsymbol{q}}^{\dagger}$, are zero within the leading order.

* naoya.arakawa@sci.toho-u.ac.jp

1 A. J. Leggett, Rev. Mod. Phys. 73, 307 (2001). 
2 A. Einstein, Sitzungsberichte der Preussischen Akademie der Wissenschaften, Physikalisch-mathematische Klasse 261 (1924); A. Einstein, Sitzungsberichte der Preussischen Akademie der Wissenschaften, Physikalischmathematische Klasse 3 (1925).

3 C. J. Pethick and H. Smith, Bose-Einstein Condensation in Dilute Gases (Cambridge University Press, Cambridge, England, 2002).

4 A. L. Fetter and J. D. Walecka, Quantum Theory of ManyParticle Systems (Dover Publications, Inc., New York, 2003).

5 Yu. D. Kalafati and V. L. Safonov, Pis'ma Zh. Eksp. Teor. Fiz. 50, 135 (1989) [JETP Lett. 50, 149 (1989)].

6 S. O. Demokritov, V. E. Demidov, O. Dzyapko, G. A. Melkov, A. A. Serga, B. Hillebrands, and A. N. Slavin, Nature (London) 443, 430 (2006).

7 Yu. M. Bunkov and G. E. Volovik, Phys. Rev. Lett. 98, 265302 (2007).

8 Yu. M. Bunkov, E. M. Alakshin, R. R. Gazizulin, A. V. Klochkov, V. V. Kuzmin, T. R. Safin, and M. S. Tagirov, JETP Lett. 94, 68 (2011); E. M. Alakshin, Yu. M. Bunkov, R. R. Gazizulin, A. V. Klochkov, V. V. Kuzmin, A. S. Nizamutdinov, T. R. Safin, and M. S. Tagirov, J. Phys. Conf. Ser. 324, 012006 (2011).

9 Yu. M. Bunkov, E. M. Alakshin, R. R. Gazizulin, A. V. Klochkov, V. V. Kuzmin, V. S. Lvov, and M. S. Tagirov, Phys. Rev. Lett. 108, 177002 (2012).

10 E. L. Fjærbu, N. Rohling, and A. Brataas, Phys. Rev. B
95, 144408 (2017).

11 N. Arakawa, Phys. Rev. Lett. 121, 187202 (2018).

12 P. W. Anderson, Phys. Rev. 86, 694 (1952).

13 T. Nakamura and M. Bloch, Phys. Rev. 132, 2528 (1963).

14 T. Holstein and H. Primakoff, Phys. Rev. 58, 1098 (1940).

15 I. S. Tupitsyn, P. C. E. Stamp, and A. L. Burin, Phys. Rev. Lett. 100, 257202 (2008).

16 K. Yosida, Prog. Theor. Phys. 6, 691 (1951).

17 C. Trapp and J. W. Stout, Phys. Rev. Lett. 10, 157 (1963).

18 R. Kubo, Phys. Rev. 15, 568 (1952).

19 T. Oguchi, Phys. Rev. 117, 117 (1960); T. Oguchi and A. Honma, J. Phys. Soc. Jpn. 16, 79 (1961).

${ }^{20}$ Using $n[\epsilon(\boldsymbol{q}) \pm h] \approx n[\epsilon(\boldsymbol{q})] \pm h n^{\prime}[\epsilon(\boldsymbol{q})]$, where $n^{\prime}(\epsilon)=$ $\partial n(\epsilon) / \partial \epsilon<0$, we obtain $n\left[\epsilon_{\alpha}\left(\boldsymbol{q}^{\prime}\right)\right]-\left(1+\delta_{\boldsymbol{q}^{\prime}, \boldsymbol{q}}\right) n\left[\epsilon_{\beta}\left(\boldsymbol{q}^{\prime}\right)\right] \approx$ $-\delta_{\boldsymbol{q}^{\prime}, \boldsymbol{q}} n\left[\epsilon\left(\boldsymbol{q}^{\prime}\right)\right]+\left(2+\delta_{\boldsymbol{q}^{\prime}, \boldsymbol{q}}\right) h n^{\prime}\left[\epsilon\left(\boldsymbol{q}^{\prime}\right)\right]$. This shows the existence of the critical magnetic field.

21 S. Takei, B. I. Halperin, A. Yacoby, and Y. Tserkovnyak, Phys. Rev. B 90, 094408 (2014).

22 T. Jungwirth, X. Marti, P. Wadley, and J. Wunderlich, Nat. Nanotechnol. 11, 231 (2016).

23 V. Baltz, A. Manchon, M. Tsoi, T. Moriyama, T. Ono, and Y. Tserkovnyak, Rev. Mod. Phys. 90, 015005 (2018).

24 N. N. Bogoliubov, Izv. Akad. Nauk SSSR, Ser. Fiz. 11, 77 (1947) [J. Phys. (USSR) 11, 23 (1947)].

25 A. A. Abrikosov, L. P. Gor'kov and I. E. Dzyaloshinski, Methods of Quantum Field Theory in Statistical Physics (Dover Publications, Inc., New York, 1963). 